\title{
Self-Consistent Cosmological Simulations of DGP Braneworld Gravity
}

\author{
Fabian Schmidt ${ }^{1,2}$ \\ ${ }^{1}$ Department of Astronomy \& Astrophysics, The University of Chicago, Chicago, IL 60637-1433 \\ ${ }^{2}$ Kavli Institute for Cosmological Physics, Chicago, IL 60637-1433
}

(Dated: September 16, 2009)

\begin{abstract}
We perform cosmological N-body simulations of the Dvali-Gabadadze-Porrati braneworld model, by solving the full non-linear equations of motion for the scalar degree of freedom in this model, the brane bending mode. While coupling universally to matter, the brane-bending mode has selfinteractions that become important as soon as the density field becomes non-linear. These selfinteractions lead to a suppression of the field in high-density environments, and restore gravity to General Relativity. The code uses a multi-grid relaxation scheme to solve the non-linear field equation in the quasi-static approximation. We perform simulations of a flat self-accelerating DGP model without cosmological constant. However, the type of non-linear interactions of the brane-bending mode, which are the focus of this study, are generic to a wide class of braneworld cosmologies. The results of the DGP simulations are compared with standard gravity simulations assuming the same expansion history, and with DGP simulations using the linearized equation for the brane bending mode. This allows us to isolate the effects of the non-linear self-couplings of the field which are noticeable already on quasi-linear scales. We present results on the matter power spectrum and the halo mass function, and discuss the behavior of the brane bending mode within cosmological structure formation. We find that, independently of CMB constraints, the self-accelerating DGP model is strongly constrained by current weak lensing and cluster abundance measurements.
\end{abstract}

Keywords: cosmology: theory; modified gravity; braneworld cosmology; Dark Energy

\section{INTRODUCTION}

The observed acceleration of the universe [1-5] poses a fascinating challenge to physicists and cosmologists: it points towards new physics at an unusually low energy scale $\left(\sim 10^{-3} \mathrm{eV}\right)$, or large length scale $\left(\sim 10^{3} \mathrm{Mpc}\right)$. For this reason, no natural explanation appears to exist up to now. Many attempts have been made to go beyond the minimal explanation, a cosmological constant or vacuum energy. They can be broadly classified into two categories: acceleration is due to an additional, smooth stress-energy component with negative pressure (dark energy [6]); or it is caused by gravity itself, through modifications to General Relativity (GR) on cosmological scales. While there are strong constraints on deviations from GR in the Solar System [7], gravity is remarkably weakly constrained on cosmological length scales. This provides an independent motivation for studying modified gravity models in the context of cosmology.

Smooth dark energy models have enough freedom to reproduce essentially any background expansion history of the universe. In order to distinguish modified gravity from the smooth dark energy scenario, it is thus necessary to study the growth of cosmological structure. A wealth of observables can be used for this purpose [8, 9, 9-18], e.g. weak lensing, galaxy-CMB cross-correlation, galaxy cluster abundances, and many more. However, almost all of these measurements are affected by non-linearities in the matter density field, or have most of their information on non-linear scales. All viable modified gravity models include a non-linear mechanism to restore General Relativity in high-density environments, which is necessary in order to satisfy Solar System constraints. This mechanism has to be taken into account in order to make accurate predictions for observables on non-linear scales in modified gravity. For one representative of $f(R)$ modified gravity, [19, 20] showed that the effects of this non-linear chameleon mechanism are significant for models that satisfy Solar System constraints.

One of the most popular modified gravity scenarios is the Dvali-Gabadadze-Porrati (DGP) model [21]. In this model, matter and radiation are confined to a 4-dimensional brane in a 5-dimensional bulk spacetime. While gravity propagates in five dimensions on the largest scales, it becomes 4-dimensional below a certain crossover scale $r_{c}$. On scales smaller than $r_{c}$, and when gravity is weak, DGP gravity can be described as an effective four-dimensional scalar-tensor theory [22]. The scalar field, referred to as brane bending mode, is massless but has non-linear derivative interactions which suppress the field in high-density environments, restoring GR locally and allowing DGP gravity to pass Solar System constraints.

Applied to homogeneous and isotropic cosmology, the DGP model allows for two solutions [23]. In one branch of the theory, the self-accelerating branch, the effective weakening of gravity on large scales leads to an accelerated late-time expansion of the brane, without any cosmological constant. The scalar brane-bending mode mediates a repulsive force, leading to a smaller effective gravitational constant on large scales. In the other solution, the normal branch, there is no accleration, and the brane-bending mode mediates an attractive force. In linear perturbation theory around its 
de Sitter limit, the brane bending mode in the self-accelerating DGP model has been shown to have the wrong sign in the kinetic term ("ghost") [22, 24], suggesting an instability, although the situation in the non-linear case is not clear $[25,26]$. The normal branch is free of the ghost.

Linear perturbation theory around a cosmological background in DGP has been derived in, e.g. [17, 27, 28]. In this approach, it is possible to predict the expansion history, CMB anisotropies, and the ISW effect in DGP [29]. Using this information, [30] have shown that the self-accelerating DGP model without cosmological constant, which has only one free parameter, $r_{c}$, is disfavored at the $\sim 4 \sigma$ level by current CMB and Supernova data. This is due to the earlier onset of acceleration in DGP, and the additional suppression of growth through the brane-bending mode.

While the self-accelerating branch of DGP is thus challenged by theory and observations, much work is being done on extending the DGP model to higher co-dimensions, in the context of degravitation [31, 32]. These higherdimensional models are expected to bring the expansion history close to that of $\Lambda \mathrm{CDM}$, while exhibiting a similar effective scalar-tensor regime as in the original DGP model (see also [33]). The form of the non-linear interactions of the brane-bending mode are also generic to these generalized models (see also [34]). Hence, it will be straightforward to perform simulations of these models once the expansion history and evolution of linear perturbations is worked out. In addition, the standard DGP model is able to satisfy the cosmological constraints if a cosmological constant (brane tension) is included [35].

So far, studies of the DGP model in the context of cosmology have mostly dealt with the linearized theory, valid on large scales. However, as soon as perturbations in the matter density become of order unity, the non-linear interactions of the brane bending mode become important (e.g., [36]). Hence, in order to make predictions for observables in the non-linear regime, the full non-linear equations of motion must be solved in conjunction with the evolution of the matter perturbations. Recently, perturbative approaches have been developed to extend the predictions into the quasi-linear regime [37]. Also, Khoury and Wyman [38] have used a spherically-symmetric approximation of the brane-bending mode interactions in their N-body simulation of braneworld models. In this paper, we present the results of an N-body simulation of the self-accelerating DGP model, which self-consistently solves the full non-linear equation for the brane-bending mode and its effect on the motion of particles (we compare our results with the approximation used in [38] in Section VIC). We solve the equation of motion in the quasi-static approximation, neglecting time derivatives with respect to spatial derivatives, as is usually done in N-body simulations (Section IV C presents a consistency test of this approximation).

We present results on the matter power spectrum, the halo mass function (abundance of dark matter halos), and the behavior of the brane bending mode in the cosmological context. These results can be used to strengthen constraints on the self-accelerating DGP model significantly, e.g., through observations of weak lensing and the abundance of galaxy clusters. More generally, the simulations presented here can serve as starting point for building a model of non-linear structure formation in braneworld scenarios, and for benchmarking perturbative approaches [37].

In Section II, we describe the DGP model, and present the relevant equations and analytical test cases. Section III presents the code, which is benchmarked in Section IV. The cosmological simulations are described in Section V. Results and their impact on cosmological constraints on the DGP model are presented in Section VI and Section VII. We conclude in Section VIII.

\section{DGP MODEL}

\section{A. Background evolution}

The Dvali-Gabadadze-Porrati model [21] consists of a spatially three-dimensional brane in a $4+1$ dimensional Minkowski bulk. Matter and all interactions except gravity are confined to the brane. The gravitational action consists of the five-dimensional Einstein-Hilbert action plus a term which leads to the 4D gravity limit on small scales:

$$
S_{\text {grav }}=-\frac{1}{16 \pi G^{(5)}} \int d^{5} X \sqrt{-g^{(5)}} R^{(5)}+S_{\text {boundary }}-\frac{1}{16 \pi G^{(4)}} \int d^{4} x \sqrt{-g^{(4)}}\left(R^{(4)}+\mathcal{L}_{m}\right) .
$$

Here, $X, g^{(5)}$ stand for the bulk coordinates and metric, while $x, g^{(4)}$ are the induced coordinates and metric on the brane, and $R^{(5)}, R^{(4)}$ denote the corresponding Ricci scalars. In the following, we will drop the ${ }^{(4)}$ notation where no confusion can arise. The boundary term is added to the action in order to ensure that variation with respect to $g^{(5)}$ leads to the correct five-dimensional Einstein equations (e.g., [39]).

The two gravitational constants, or Planck masses $M_{\mathrm{Pl}(5)}, M_{\mathrm{Pl}(4)}$ appearing in Eq. (2.1) can be related via a length 
scale, the crossover scale:

$$
r_{c} \equiv \frac{1}{2} \frac{G^{(5)}}{G^{(4)}}=\frac{1}{2} \frac{M_{\mathrm{Pl}(4)}^{2}}{M_{\mathrm{Pl}(5)}^{3}} .
$$

On scales above $r_{c}$, gravity becomes five-dimensional, with forces falling off as $1 / r^{3}$. Below $r_{c}$, gravity is fourdimensional, but not Einsteinian gravity, a point to which we return below.

Since all matter is thought as confined to the brane, the five-dimensional metric has to obey non-trivial junction conditions over the brane [23]. Assuming an empty Minkowski bulk, a spatially flat brane, and a homogeneous and isotropic matter distribution on the brane, the junction conditions lead to the following analogue of the Friedmann equation for the scale factor of the induced metric on the brane:

$$
H^{2} \pm \frac{H}{r_{c}}=\frac{8 \pi G}{3} \rho, \quad H \equiv \frac{\dot{a}}{a}
$$

The junction conditions leave two possible branches of the theory, determined by the sign on the left hand side of Eq. (2.3). In the following, we focus on the branch with the '-' sign, which asymptotes to a late-time de SitterUniverse, $H=1 / r_{c}=$ const., and is correspondingly called the accelerating branch. Then, in the matter-dominated epoch, neglecting radiation, and again assuming no cosmological constant or curvature, Eq. (2.3) can be rewritten as:

$$
\begin{aligned}
E(a) \equiv \frac{H(a)}{H_{0}} & =\sqrt{\Omega_{\mathrm{rc}}}+\sqrt{\Omega_{m} a^{-3}+\Omega_{\mathrm{rc}}}, \text { where } \\
\Omega_{\mathrm{rc}} & \equiv \frac{1}{4 H_{0}^{2} r_{c}^{2}} ; \quad \Omega_{m} \equiv \frac{8 \pi G}{3 H_{0}^{2}} \rho_{m 0},
\end{aligned}
$$

and $\rho_{m 0}$ is the average matter density today. This expansion history is clearly different from $\Lambda$ CDM, and corresponds to an effective dark energy with $w_{\text {eff }} \rightarrow-1 / 2$ in the matter-dominated era at high redshifts.

\section{B. Cosmological perturbations and brane bending mode}

The propagation of light and particles on the DGP brane is completely determined by the perturbed 4D FriedmannRobertson-Walker metric:

$$
d s^{2}=-[1+2 \Psi(\mathbf{x}, t)] d t^{2}+[1+2 \Phi(\mathbf{x}, t)] a^{2}(t) d \mathbf{x}^{2}
$$

However, in order to determine the evolution of the metric potentials on the brane, it is necessary to solve the full 5D Einstein equations $[17,36]$. An additional scalar degree of freedom associated with local displacements of the brane appears, the so-called brane-bending mode $\varphi$ which couples to matter. In our convention, $\varphi$ is dimensionless, instead of being scaled to the Planck mass $M_{\mathrm{Pl}(4)}$.

In the decoupling limit of DGP [22], when setting gravitational interactions to 0 , the self-interactions of the $\varphi$ field remain constant. Hence, while perturbations of the metric higher than linear order can be neglected for cosmological studies, it is crucial to consider the self-interactions in the brane-bending mode.

In the quasi-static regime, for scales $k \gg H_{0}, r_{c}^{-1}$, time derivatives can be neglected with respect to spatial derivatives, and the equation for the brane-bending mode reads (e.g., [36]):

$$
\nabla^{2} \varphi+\frac{r_{c}^{2}}{3 \beta a^{2}}\left[\left(\nabla^{2} \varphi\right)^{2}-\left(\nabla_{i} \nabla_{j} \varphi\right)\left(\nabla^{i} \nabla^{j} \varphi\right)\right]=\frac{8 \pi G a^{2}}{3 \beta} \delta \rho,
$$

where the derivatives are with respect to comoving coordinates $\mathbf{x}, \delta \rho=\rho_{m}-\overline{\rho_{m}}$ is the matter density perturbation, and the function $\beta(a)$ (for the accelerating branch) is given by:

$$
\beta(a)=1-2 H(a) r_{c}\left(1+\frac{\dot{H}(a)}{3 H^{2}(a)}\right) .
$$

Note that $\beta$ is always negative in the self-accelerating branch. In Section IV C, we show results of a consistency test of the quasi-static assumption used in Eq. (2.6).

The brane-bending mode influences the dynamics of particles through the dynamical potential $\Psi$, which, assuming the same boundary conditions for $\Psi$ and $\varphi$, is given by:

$$
\Psi=\Psi_{N}+\frac{1}{2} \varphi
$$


where the Newtonian potential $\Psi_{N}$ satisfies the usual Poisson equation:

$$
\nabla^{2} \Psi_{N}=4 \pi G a^{2} \delta \rho
$$

In contrast, the propagation of photons, determined by the lensing potential $\Phi_{-} \equiv(\Phi-\Psi) / 2$, is not directly affected by $\varphi$.

\section{Weak brane regime}

If the gradient of the $\varphi$ field is small, i.e. for small gravitational accelerations, Eq. (2.6) can be linearized, yielding a standard Poisson equation:

$$
\nabla^{2} \varphi_{L}=\frac{8 \pi G a^{2}}{3 \beta} \delta \rho \Rightarrow \varphi_{L}=\frac{2}{3 \beta} \Psi_{N}
$$

We will refer to a cosmology with this linearized equation as the linearized DGP model. In this regime, also called weak-brane phase, $\varphi=\varphi_{L}$ becomes proportional to the Newtonian potential $\Psi_{N}$, corresponding to a constant rescaling of the gravitational constant through Eq. (2.8):

$$
G_{N} \rightarrow G_{\mathrm{eff}}=\left(1+\frac{1}{3 \beta}\right) G_{N}
$$

Substituting the linear solution into Eq. (2.6), we obtain a rough estimate for the overdensity $\delta \equiv \delta \rho / \bar{\rho}_{m}$ at which the non-linear interactions become important:

$$
1 \sim \frac{r_{c}^{2}}{3 \beta a^{2}}\left(\nabla^{2} \varphi_{L}\right)^{2} / \nabla^{2} \varphi_{L}=\frac{8 \pi G r_{c}^{2}}{9 \beta^{2}} \delta \rho=\frac{3 H_{0}^{2} r_{c}^{2}}{9 \beta^{2}} \Omega_{m} \delta
$$

For a self-accelerating DGP model that leads to cosmic acceleration today, $r_{c} \sim H_{0}^{-1}, \beta(a=1) \sim-1$, and the prefactor in Eq. (2.12) is of order unity today. Hence, the self-coupling of the brane bending mode $\varphi$ becomes important as soon as the matter density field becomes non-linear, $\delta \sim 1$.

\section{Vainshtein effect}

In general, Eq. (2.6) is difficult to solve in full generality due to the non-linearity in the derivative terms. However, an instructive test case, a spherically symmetric matter distribution, can be solved analytically. In the spherically symmetric case, Eq. (2.6) becomes [36]:

$$
\begin{gathered}
\left(\frac{d^{2}}{d r^{2}}+\frac{2}{r} \frac{d}{d r}\right)\left(\varphi+\frac{1}{3 \beta a^{2}} \Xi\right)=\frac{8 \pi G a^{2}}{3 \beta} \delta \rho, \\
\Xi \equiv 2 r_{c}^{2} \int_{0}^{r} \frac{d r^{\prime}}{r^{\prime}}\left(\frac{d \varphi}{d r^{\prime}}\right)^{2} .
\end{gathered}
$$

For simplicity, we assume a spherical mass $M$ of radius $R$ with uniform density, and set $a=1$. Then, we can integrate Eq. (2.13) once and obtain the gravitational acceleration in DGP:

$$
g=g_{N}+\frac{1}{2} \frac{d \varphi}{d r}=g_{N}[1+\Delta(r)]
$$

where $g_{N}$ is the Newtonian acceleration of the spherical mass, and:

$$
\Delta(r)=\frac{2}{3 \beta}\left\{\begin{array}{l}
r^{3} / r_{*}^{3}\left(\sqrt{1+\left(\frac{r_{*}}{r}\right)^{3}}-1\right), r \geq R \\
R^{3} / r_{*}^{3}\left(\sqrt{1+\left(\frac{r_{*}}{R}\right)^{3}}-1\right), r<R .
\end{array}\right.
$$


Here, $r_{*}$ denotes a characteristic scale of the solution, the Vainshtein radius:

$$
r_{*}^{3}=\frac{8 r_{c}^{2} r_{s}}{9 \beta^{2}},
$$

and $r_{s}=2 G M$ is the gravitational radius of the mass. For very large distances, $r \gg r_{*}, \Delta(r)$ approaches the constant $1 /(3 \beta)$, which exactly matches the linear solution, Eq. (2.11). Note also that by substituting $\delta \rho \sim M / r^{3}$ in Eq. (2.12), we see that the non-linearity criterion is directly proportional to $\left(r_{*} / r\right)^{3}$.

In the opposite limit, $r \ll r_{*}, \varphi$ becomes suppressed with respect to the linear solution, and $\Delta(r)$ approaches the small constant $2 /(3 \beta)\left(R / r_{*}\right)^{3 / 2}$ inside the mass (assuming $R \ll r_{*}$ ). This Vainshtein effect [40, 41] amounts to restoring GR in deep potential wells, while far away from the mass, gravity is in the scalar-tensor regime.

Fig. 1 shows the analytical solution for the $\varphi$ field and the deviation from the Newtonian acceleration, as well as the code results which agree well with the analytical solution in the regime of validity (see Section IV A).

\section{E. Plane wave solution}

Another exact solution to Eq. (2.6) exists: for a simple plane wave density perturbation, $\delta \rho(\mathbf{x})=A \exp (i \mathbf{k} \cdot \mathbf{x})$. In this case, the two non-linear terms exactly cancel, and we are left with the linear solution, Eq. (2.10). In Appendix B this is used to test our code for perturbations of different wavelengths. Also, the plane wave and spherically symmetric solutions can be considered as two limiting cases for understanding the $\varphi$ field behavior in the cosmological context. We will return to this point in Section VI C.

\section{CODE IMPLEMENTATION}

We use an extension of the code first described in [42] for our simulations of DGP gravity. The code is a standard particle-mesh N-body code $[43,44]$ assuming collisionless dark matter only, with a fixed grid of size $N_{g}^{3}$ and periodic boundary conditions, and uses second-order accurate leapfrog integration for the particle propagation. The major addition is a relaxation solver for the non-linear equation of the brane bending mode $\varphi$, Eq. (2.6). The simulation proceeds by performing steps $\Delta a$ in the scale factor $a$.

Except for the different expansion history, the equations for particle propagation are identical to those in standard CDM simulations, and are expressed in terms of comoving coordinates $\mathbf{x}, \mathbf{p}$ :

$$
\begin{aligned}
\frac{d \mathbf{x}_{i}}{d a} & =\frac{1}{a^{3} E(a)} \mathbf{p}_{i}, \\
\frac{d \mathbf{p}_{i}}{d a} & =-\frac{1}{a E(a)} \nabla \Psi\left(\mathbf{x}_{i}\right) .
\end{aligned}
$$

Here, $\mathbf{x}_{i}$ and $\mathbf{p}_{i}$ are the position and momentum of particle $i$ in code units (see Appendix A), and $a E(a)$ is taken from Eq. (2.4). The main modification enters in the determination of $\Psi(\mathbf{x})$. One time step of the N-body simulation proceeds as follows:

- The density field on a fixed grid is assigned using the particle positions at scale factor $a$ via the cloud-in-cell method, i.e. each particle corresponds to a uniform density cube of side length $r_{\text {cell }}$ and mass $M=\bar{\rho}_{m} r_{\text {cell }}^{3}$.

- The Newtonian potential $\Psi_{N}$ is solved for using a Fast Fourier Transform; Eq. (2.6) is solved via relaxation, and the overall dynamical potential $\Psi$ is assembled according to Eq. (2.8). We also run linearized DGP simulations, where the potential is simply given by $\Psi=[1+1 /(3 \beta(a))] \Psi_{N}$.

- Using the same cloud-in-cell interpolation as for particles, the gradient of $\Psi$ is calculated at each particle position and is used to update the particle momenta from $a-\Delta a / 2$ to $a+\Delta a / 2$.

- Using the momenta at $a+\Delta a / 2$, the particle positions are updated from $a$ to $a+\Delta a$, and the process starts from the beginning.

Thus, in each time step we have to solve for the brane-bending mode $\varphi$ which contributes to the dynamical potential $\Psi$ via Eq. (2.8). In other words, given the density field, we have to solve the non-linear elliptical differential equation Eq. (2.6). We use a Gauss-Seidel relaxation scheme together with the Newton method. A crucial tool is the multigrid [45-47], a hierarchy of coarser grids which are essential to speeding up the convergence [42]: as the relaxation scheme, 

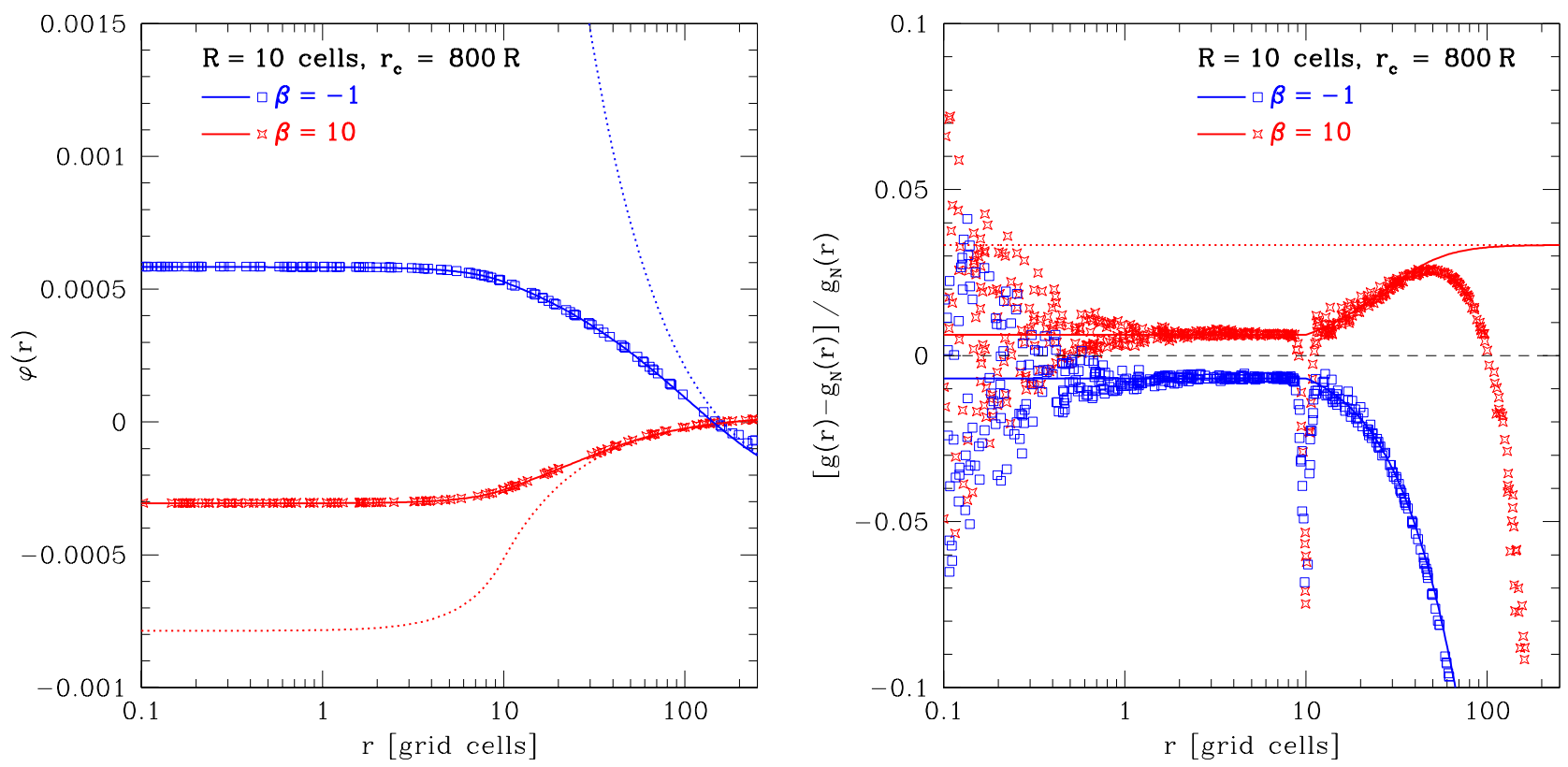

FIG. 1: Left panel: $\varphi(r)$ measured in simulations with a $512^{3}$ grid for a spherical mass for different values of $\beta$, where $r$ is the distance from the center of the mass. $R=10$ grid cells, and $r_{c}=800 R$ is held fixed (see text). The solid lines show the full analytical solution for the spherical mass, while dotted lines show the linearized solution (Section II C). Right panel: Relative deviation of the acceleration measured in the spherical mass solution from the analytical Newtonian value vs. $r$, for the same parameters as in the left panel. Again, solid lines show the full exact solution, while dotted lines show the linearized solution.

which operates locally, gets rid of small-scale errors very efficiently, the coarser grids quickly reduce the long-wavelength error modes which are hard to eliminate on the fine grid alone. For details of the implementation, see Appendix A.

While the $\varphi$ field is in general well-behaved, the convergence properties become worse for strongly inhomogeneous density fields, because the non-linearities in Eq. (2.6) are in the derivatives of the $\varphi$ field. In order to reach the desired convergence in our cosmological simulations, it is necessary to smooth the density field entering the r.h.s. of Eq. (2.6) with a Gaussian kernel $\propto \exp \left(-r^{2} / 2 r_{s}^{2}\right)$, with $r_{s}$ set to the size of a grid cell. This smoothes over the noise in the density field due to the discreteness of particles.

\section{CODE TESTS}

In this section, we present different tests our code was put through to benchmark its performance and limitations. We focus on tests applying to the modified gravity sector. For the results of standard N-body code tests for this code, see [42].

\section{A. Spherical mass test}

In order to study how well the code reproduces the exact result for a spherical mass in DGP, we start out with a grid with $N_{g}^{3}$ grid cells and $N_{P}$ particles. We arbitrarily assume a box size of $L_{\mathrm{box}}=200 \mathrm{Mpc} / h$ and set $a=1$. All particles are moved into a spherical mass of radius $R$ and uniform density, which then corresponds to a mass of $M=\rho_{m 0} L_{\text {box }}^{3} \approx 7 \cdot 10^{17} M_{\odot} / h$ (assuming $\Omega_{m}=0.3$ ). Given the density field assigned from the particle positions, we then use the relaxation solver to solve for $\varphi$, and measure the field values and radial acceleration throughout the box.

For the purposes of this test, we can vary the two parameters $\beta$ and $r_{c}$ in Eq. (2.6) independently. $1 / \beta$ determines the strength of the additional force mediated by $\varphi$ [e.g., Eq. (2.10)], which is attractive for positive $\beta$ and repulsive for $\beta<0$. For given $\beta, r_{c}$ controls the Vainshtein radius, i.e. the scale where the self-interactions of the $\varphi$ field become important.

Fig. 1 shows the field solution (left panel) and relative deviation of the acceleration from the Newtonian value (right panel) as a function of distance from the center, $r$, for $N_{g}=512, R=10$ grid cells, corresponding to $3.9 \mathrm{Mpc} / h$, 

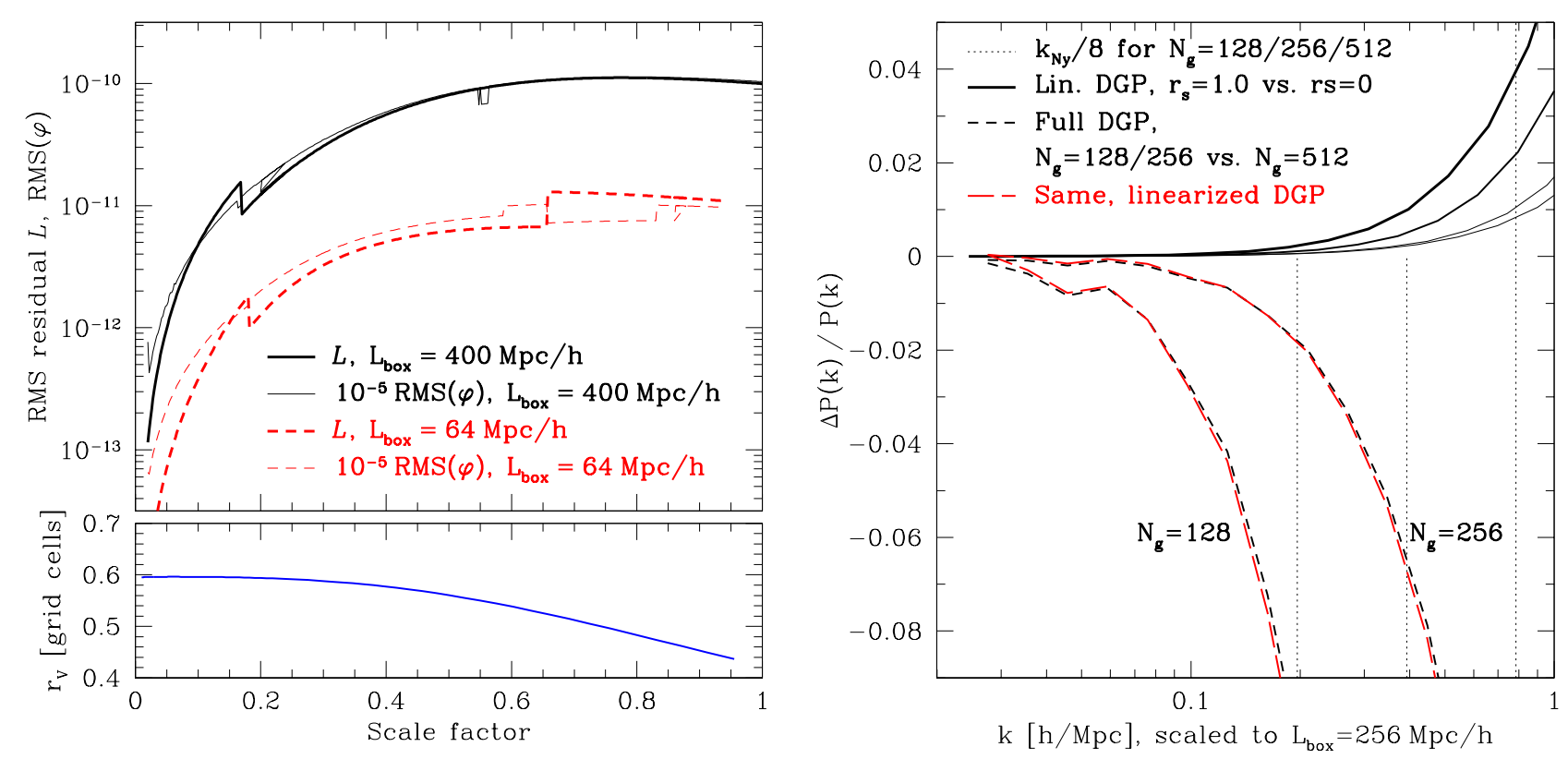

FIG. 2: Left panel: Dimensionless RMS residual $\mathcal{L}=\sqrt{\left\langle r^{2}\right\rangle}$ and RMS of the $\varphi$ field vs. $a$ in cosmological simulations (top), and Vainshtein radius for a single particle in the simulations, in units of $r_{\text {cell }}$ (bottom). Right panel: Relative deviation of linearized DGP power spectra smoothed with $r_{s} / r_{\text {cell }}=1.0$ from the unsmoothed simulations (solid lines, for box sizes from $64 \mathrm{Mpc} / h$ [thick] to $400 \mathrm{Mpc} / h$ [thin]), and relative deviation of power spectra for lower resolution simulations $\left(N_{g}=128\right.$, left dashed curves, $N_{g}=256$, right dashed curves). Also shown as vertical dotted lines are the maximum wavenumbers considered for each simulation, $k_{\max }=k_{\mathrm{Ny}} / 8$.

and $r_{c} / R=800 \approx 3100 \mathrm{Mpc} / h$. The agreement with the analytical solution (thick lines) is generally very good, except at large radii, where the periodic boundary conditions become important, and around $r=R$, where artifacts of interpolating a sphere onto a cubic grid become visible. At distances of order the size of the grid cells, the force resolution becomes worse leading to a growing scatter in the measured acceleration. Note that the analytical solution necessarily assumes an isolated mass, since the superposition principle cannot be used due to the non-linear $\varphi$ field equation. For this reason, we added an arbitrary zero-point to the $\varphi$ field in order to match the analytical solution in the left panel of Fig. 1. Of course, such a zero-point does not affect any observable quantity, such as the acceleration shown in the right panel.

We tested the field solution for several different values of $R, N_{g}, \beta$, and $r_{c}$. Given the caveats pointed out, we found very good convergence to the analytical solution. In particular, increasing $R$ reduces the interpolation artifacts at $r=R$, while decreasing $R$ reduces the impact of the periodic boundary conditions. Solutions for different values of $\beta$ are shown in both panels of Fig. 1. While the solid lines show the exact solution to the full equation, Eq. (2.13), the dotted lines show the linearized result, Eq. (2.10). Clearly, for the parameters chosen, the effect of the non-linearity is significant for $r$ of a few $R$ or less.

\section{B. Convergence and resolution tests}

In most test cases we studied, the density field is sufficiently smooth that the relaxation converges to the desired low tolerance. However, for density fields with considerable small-scale inhomogeneities, such as the cosmological density field at late times, we find that this level is not achievable. This is because the non-linearity of the $\varphi$ equation is important as soon as the overdensity $\delta$ becomes of order unity, which in the N-body simulation corresponds to \pm 1 particle per grid cell. Equivalently, the Vainshtein radius for a single particle in our N-body simulation is of order the grid scale (see Fig. 2, bottom left). Thus, the $\varphi$ field reacts much more strongly to noise due to the discreteness of particles than the Newtonian potential, leading to increased residual errors of the approximate solution.

If we write Eq. (2.6) in code units (see Appendix A) as $L(\varphi)=f$, then for an approximate solution $\varphi_{i}, r \equiv$ $r_{\text {cell }}^{2}\left(L\left(\varphi_{i}\right)-f\right)$ is the dimensionless residual, where $r_{\text {cell }}=L_{\mathrm{box}} / N_{g}$ is the size of a grid cell. In the following, we use the RMS of the dimensionless residual, $\mathcal{L} \equiv \sqrt{\left\langle r^{2}\right\rangle}$ as a benchmark for the convergence of the field solution. 
We performed tests with sine wave density fields of various wavelengths in order to determine what residual $\mathcal{L}$ is acceptable in our simulations (see Appendix B). In case of a pure sine wave, the non-linearity in Eq. (2.6) vanishes, and the exact non-linear solution is identical to the linearized solution. We found that residuals of $\mathcal{L} \lesssim 10^{-10}$ are safe, as for residuals at that level, the errors in the solution are negligible compared to the unavoidable truncation errors from taking numerical derivatives on the grid.

In order to reduce the noise in the density field in the cosmological simulations and improve the solution to the acceptable level of $\mathcal{L}$, we increase the number of particles from $\left(N_{g} / 2\right)^{3}$ to $N_{g}^{3}$, and smooth the density field entering the r.h.s. of Eq. (2.6) as described in Section III. With these steps, the solution converges with a residual $\mathcal{L} \lesssim 10^{-10}$ for all box sizes (left panel of Fig. 2). Note that the dimensionless RMS residuals never exceed a fraction of $\sim 10^{-5}$ of the RMS of the $\varphi$ field solution.

Since the force resolution in our simulations is in any case limited to scales above $r_{\text {cell }}$, a smoothing on the grid scale is not expected to degrade the resolution of the simulations significantly. We checked this by running linearized DGP simulations given by Eq. (2.10) (Section II C) using the same smoothing of the r.h.s. of Eq. (2.6), and comparing the resulting power spectrum to that of linearized DGP simulations without smoothing, for the same initial conditions. The result is shown in the right panel of Fig. 2. As expected, the effect of smoothing increases towards larger $k$. The smoothing effect on the matter power spectrum is positive, since the smoothing removes power in the $\varphi$ field on the smallest scales, and $\varphi$ mediates a repulsive force. This leads to an enhancement in the matter power spectrum.

The smoothing effect remains below $4 \%$ for $k<k_{\max }=k_{\mathrm{Ny}} / 8$, which we adopt as the maximum wave number considered for each box, where $k_{\mathrm{Ny}}=\pi N_{g} / L_{\mathrm{box}}$ is the Nyquist frequency of the grid. From our studies with different smoothing radii $r_{s}=0.8 \ldots 3.0$, we found that the smoothing effects on full DGP simulations show a similar $k$ dependence as those of the linearized DGP simulations, but are smaller by a factor of $\sim 0.6$. This is understandable since the $\varphi$ field is suppressed in dense regions in the full simulations, reducing the effect of the smoothing. Taking into account this factor, we correct the power spectra measured in the full DGP simulations for the smoothing effects using the curves shown in Fig. 2 (right panel). Note that in any case these effects are at the level of few percent or less.

In order to assess the effect of the finite grid resolution, we also performed simulations with $N_{g}=256$ and $N_{g}=128$, and $N_{p}=N_{g}^{3}$. Fig. 2 (right panel) also shows the deviation of the power spectrum in these low-resolution simulations from the $N_{g}=512$ simulation with the same initial conditions. The vertical lines indicate $k_{\max }=k_{\mathrm{Ny}} / 8$ for each case, i.e. the maximum $k$ we consider for each simulation box. Below $k_{\mathrm{Ny}} / 8$, the deviations are less than $10 \%$ for either $N_{g}=256$ or $N_{g}=128$. Note also that the resolution effects are independent of the type of simulation (DGP, linearized DGP, or GR). Hence, they cancel when measuring the deviation of $P(k)$ between different simulation types, and we expect this deviation to be accurate to the percent level for the range in $k$ we consider.

\section{Quasi-static approximation}

In solving for the brane bending mode $\varphi$, we have assumed that all time-derivative terms in the full equation of motion of $\varphi$ (in terms of physical coordinates):

$$
\square \varphi+\frac{r_{c}^{2}}{3 \beta}\left[(\square \varphi)^{2}-\left(\nabla_{\mu} \nabla_{\nu} \varphi\right)^{2}\right]=\frac{8 \pi G}{3 \beta} \delta \rho,
$$

can be neglected with respect to the spatial derivatives in Eq. (2.6). Up to second order, there are three time derivative terms which have been neglected:

$$
\begin{aligned}
& T_{0}=a^{2} \partial_{t t} \varphi \\
& T_{1}=\frac{2 r_{c}^{2}}{3 \beta} \partial_{t t} \varphi \cdot \nabla^{2} \varphi \\
& T_{2}=\frac{2 r_{c}^{2}}{3 \beta}\left(\partial_{t} \nabla_{i} \varphi\right)^{2} .
\end{aligned}
$$

$T_{1}$ and $T_{2}$ appear as $T_{1}-T_{2}$ in the equations of motion. While we cannot rigorously prove that the quasi-static approximation holds, we can perform a consistency test by measuring the terms Eqs. (4.2)-(4.4) in the simulations, and checking whether they are indeed small. The time derivatives of $\varphi$ are calculated in each grid cell using the two neighboring time steps. We then take the RMS of each term over the grid, $\left\|T_{i}\right\|=\sqrt{\left\langle T_{i}^{2}\right\rangle}$, and compare with the RMS of the spatial Laplacian, $\left\|\nabla^{2} \varphi\right\|$. Fig. 3 presents $T_{0}$ and $T_{1}-T_{2}$ relative to the spatial derivatives for our largest and smallest box sizes, and shows that they are well under control. The relative magnitude of all time derivatives are of order a few $10^{-5}$ or less. This is in keeping with the expectation that $\partial \varphi / \partial t \sim H \varphi$, and $k / a \gg H$ in our simulations. We conclude that the quasi-static approach is a self-consistent approximation. 


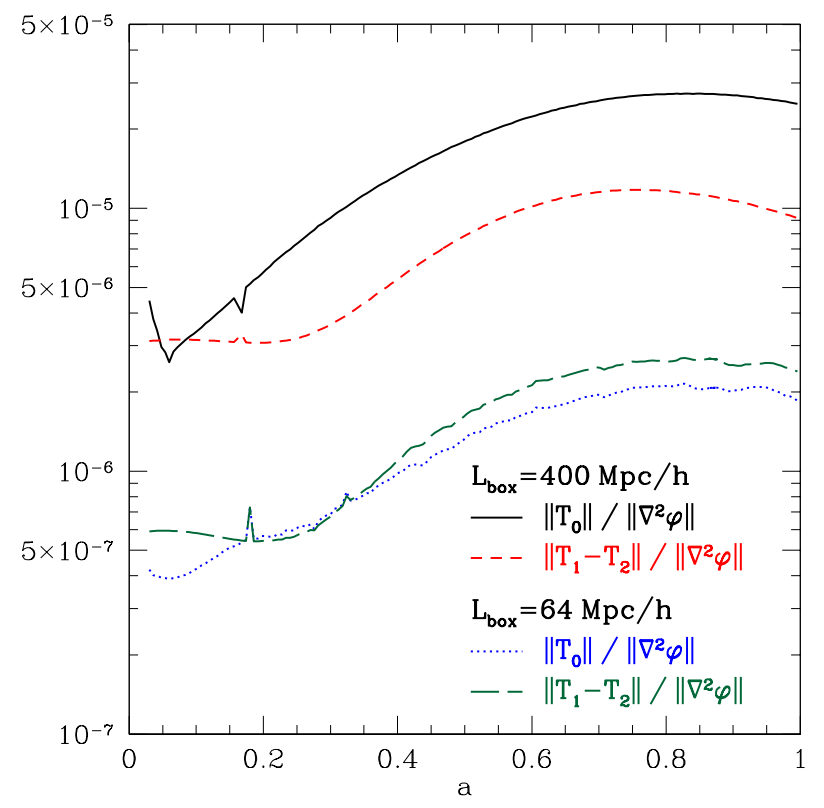

FIG. 3: The time derivative terms Eqs. (4.2)-(4.4) of $\varphi$ measured in the simulations relative to the spatial Laplacian, as a function of scale factor $a$ for our largest and smallest simulation boxes. The time derivatives always remain $5-6$ orders of magnitude below the spatial derivatives.

\section{COSMOLOGICAL SIMULATIONS}

We performed a suite of cosmological simulations for three different types of gravity: unmodified GR, corresponding to a smooth dark energy model with the same expansion history as DGP, referred to as QCDM; linearized DGP gravity, using the linearized $\varphi$ equation of motion [Eq. (2.10)], corresponding to a time-dependent rescaling of Newton's constant; and full DGP, solving for the full non-linear $\varphi$ solution [Eq. (2.6)]. Comparing linearized DGP with full DGP allows us to study the Vainshtein effect in a cosmological setting.

The cosmological parameters are those of the best-fit flat self-accelerating DGP model to WMAP 5yr data [48] and are summarized in Tab. I. We generated the initial conditions at $a=0.02(z=49)$ from a modified transfer function output of CAMB [49] for a flat $\Lambda$ CDM model with $\Omega_{\Lambda}=1-\Omega_{m}$. The $\Lambda$ CDM transfer function was corrected for small early-time modified gravity effects in the DGP model using the PPF approach, as detailed in Appendix C.

The simulations were run with $N_{g}=512$ grid cells on a side, and $N_{p}=512^{3}$ particles, i.e. one particle per grid cell, to reduce the shot noise in the density field (see Section IVB). We performed six simulations each for four different box sizes, from $L_{\mathrm{box}}=64 \mathrm{Mpc} / h$ up to $L_{\mathrm{box}}=400 \mathrm{Mpc} / h$ (Tab. II). On an 8-core machine, the QCDM and linearized

TABLE I: Cosmological parameters.

\begin{tabular}{l|l}
\hline$\Omega_{m}$ & 0.258 \\
$\Omega_{\mathrm{rc}}$ & 0.138 \\
$H_{0}$ & $66.0 \mathrm{~km} / \mathrm{s} / \mathrm{Mpc}$ \\
\hline $100 \Omega_{b} h^{2}$ & 2.37 \\
$\Omega_{c} h^{2}$ & 0.0888 \\
$\tau$ & 0.0954 \\
$n_{s}$ & 0.998 \\
$A_{s}\left(k=0.05 \mathrm{Mpc}^{-1}\right)$ & $2.01610^{-9}$ \\
\hline \hline$\sigma_{8}(\Lambda \mathrm{CDM})$ & $0.6566^{a}$ \\
\hline
\end{tabular}

${ }^{a}$ Linear power spectrum normalization today of a $\Lambda$ CDM model with the same primordial normalization.
TABLE II: Simulation type and number of runs per box size.

\begin{tabular}{|c|c|c|c|c|c|}
\hline & \multicolumn{4}{|c|}{$L_{\text {box }}[\mathrm{Mpc} / h]$} \\
\hline & & 400 & 256 & 128 & 64 \\
\hline \multirow{3}{*}{$\begin{array}{c}\# \text { of } \\
\text { boxes }\end{array}$} & QCDM & 6 & 6 & 6 & 6 \\
\hline & Lin. DGP & 6 & 6 & 6 & 6 \\
\hline & Full DGP & 6 & 6 & 6 & 6 \\
\hline \multicolumn{2}{|c|}{$k_{\max }=k_{\mathrm{Ny}} / 8[h / \mathrm{Mpc}]$} & 0.50 & 0.79 & 1.57 & 3.14 \\
\hline \multicolumn{2}{|c|}{$r_{\text {cell }}[\mathrm{Mpc} / h]$} & 0.78 & 0.50 & 0.25 & 0.13 \\
\hline \multicolumn{2}{|c|}{$M_{\min }\left[10^{12} M_{\odot} / h\right]$} & 219 & 57.3 & 7.17 & 0.90 \\
\hline
\end{tabular}



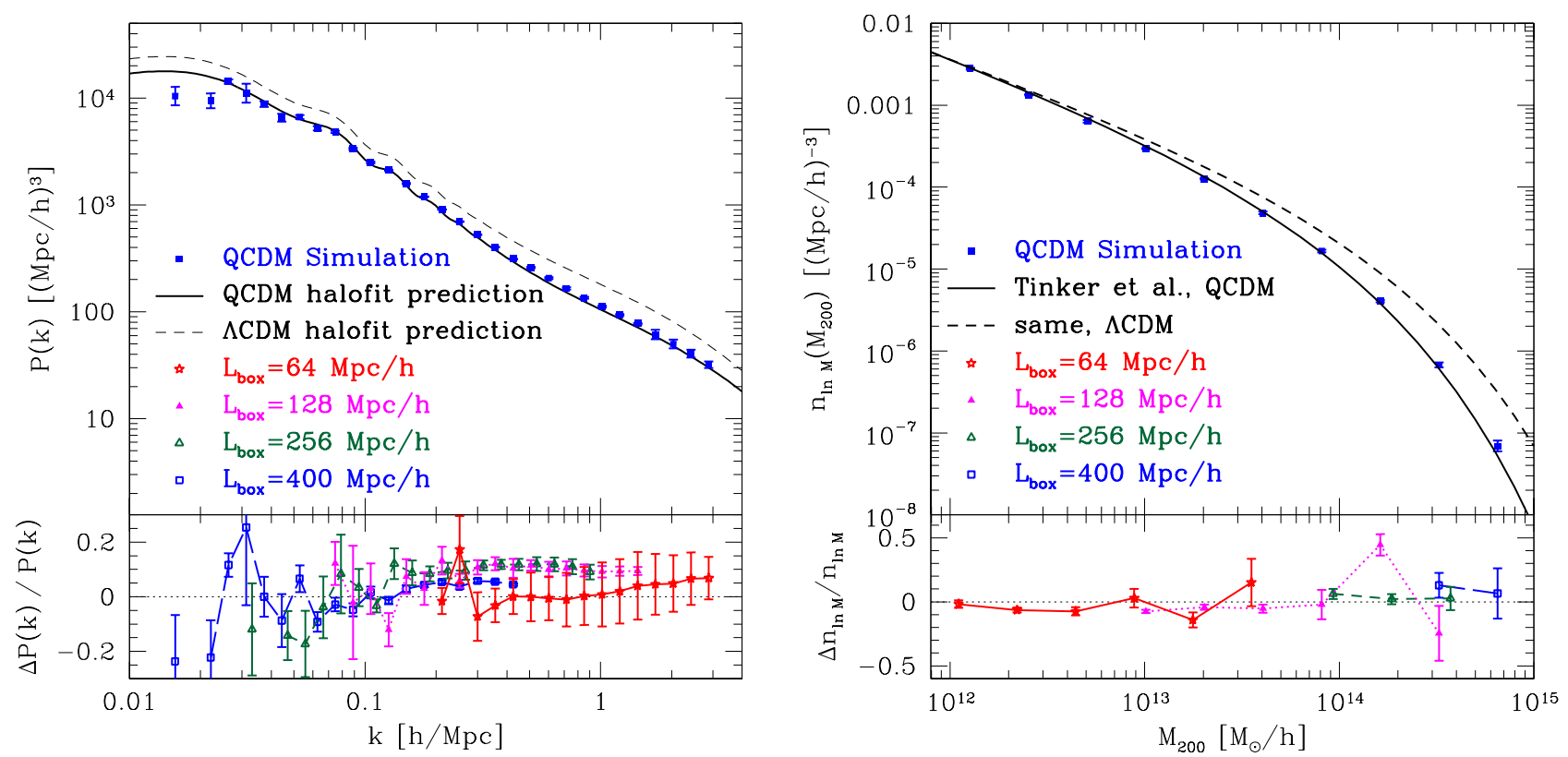

FIG. 4: Left panel: Power spectrum measured in the QCDM effective dark energy cosmology simulations (top), and halofit predictions. The lower panel shows the deviations from the halofit prediction separately for each simulation box size. Right panel: Halo mass function $d n / d \ln M_{200}$ measured in the QCDM simulations (points) and the mass function prediction for QCDM after [51]. Also shown is the mass function for a $\Lambda$ CDM model with the same primordial power spectrum. The lower panel shows the deviation from the prediction separately for each box size.

DGP runs require $\sim 10 \mathrm{~h}$ of computing time, while the full DGP simulations require about $300 \mathrm{~h}$.

Fig. 4 (left panel) shows the combined power spectrum from all box sizes for the QCDM simulations, including bootstrap errors. Also shown is the non-linear power spectrum for QCDM, calculated from the linear power spectrum using the halofit procedure [50]. The power spectrum measured in each box is used up to $k_{\max }=k_{\mathrm{Ny}} / 8$ (see Tab. II), and different boxes are combined weighting by volume. The lower panel of Fig. 4 (left) shows the power spectrum relative to the halofit prediction, measured separately for each box size. The power spectra measured in different boxes clearly agree within the errors, and the deviations from the halofit prediction are within the accuracy $(\sim 20 \%)$ expected from this fitting procedure (especially given the significant departures from $\Lambda$ CDM of the simulated expansion history).

Fig. 4 also shows the non-linear power spectrum for a flat $\Lambda \mathrm{CDM}$ cosmology fixed to the same $\Omega_{m}, h$, and primordial normalization, corresponding to a linear normalization of $\sigma_{8}=0.66$ today. Note that due to the different expansion history and the earlier onset of acceleration in DGP, the linear growth factor is suppressed by $\sim 15 \%$ in QCDM relative to $\Lambda \mathrm{CDM}$. The repulsive brane-bending mode in DGP suppresses growth further, leading to a suppression in the linear regime of $\sim 21 \%$ relative to $\Lambda \mathrm{CDM}$. In the following, we will always compare the DGP results with QCDM, so that the expansion history effects are taken out, and all deviations are strictly due to modifications of gravity.

We have also measured the mass function of dark matter halos in our simulations. The spherical overdensity halofinding and combination of different simulation boxes was done as described in [20]. As our grid resolution is the same as in the simulations analyzed in [20], while we have a factor of 8 more particles, we conservatively increase the minimum required number of particles from 800 to 6400 . The corresponding mass thresholds are given in Tab. II. We define the halo mass $M_{200}$ as the mass enclosed within a sphere of radius $R_{200}$, so that the average density within the sphere is 200 times the average matter density in the universe, $\bar{\rho}_{m}=\Omega_{m} \rho_{\text {crit }}$.

Fig. 4 (right panel) shows the mass function, $n_{\ln M} \equiv d n / d \ln M_{200}$, measured in QCDM simulations, in comparison with the prediction calculated from the linear QCDM power spectrum using the fitting formula from Tinker et al. [51]. The lower panel shows the relative deviations from the prediction, separately for each simulation box. Within the mass range accessible with our simulations, $M_{200} \sim 10^{12}-10^{15} M_{\odot} / h$, the agreement with the prediction and among different boxes is good. We also show the predicted mass function for a $\Lambda$ CDM model with the same initial power spectrum in Fig. 4. Apparently, the number of halos above $\sim 10^{14} M_{\odot} / h$ is significantly reduced due to the suppressed growth in QCDM. 

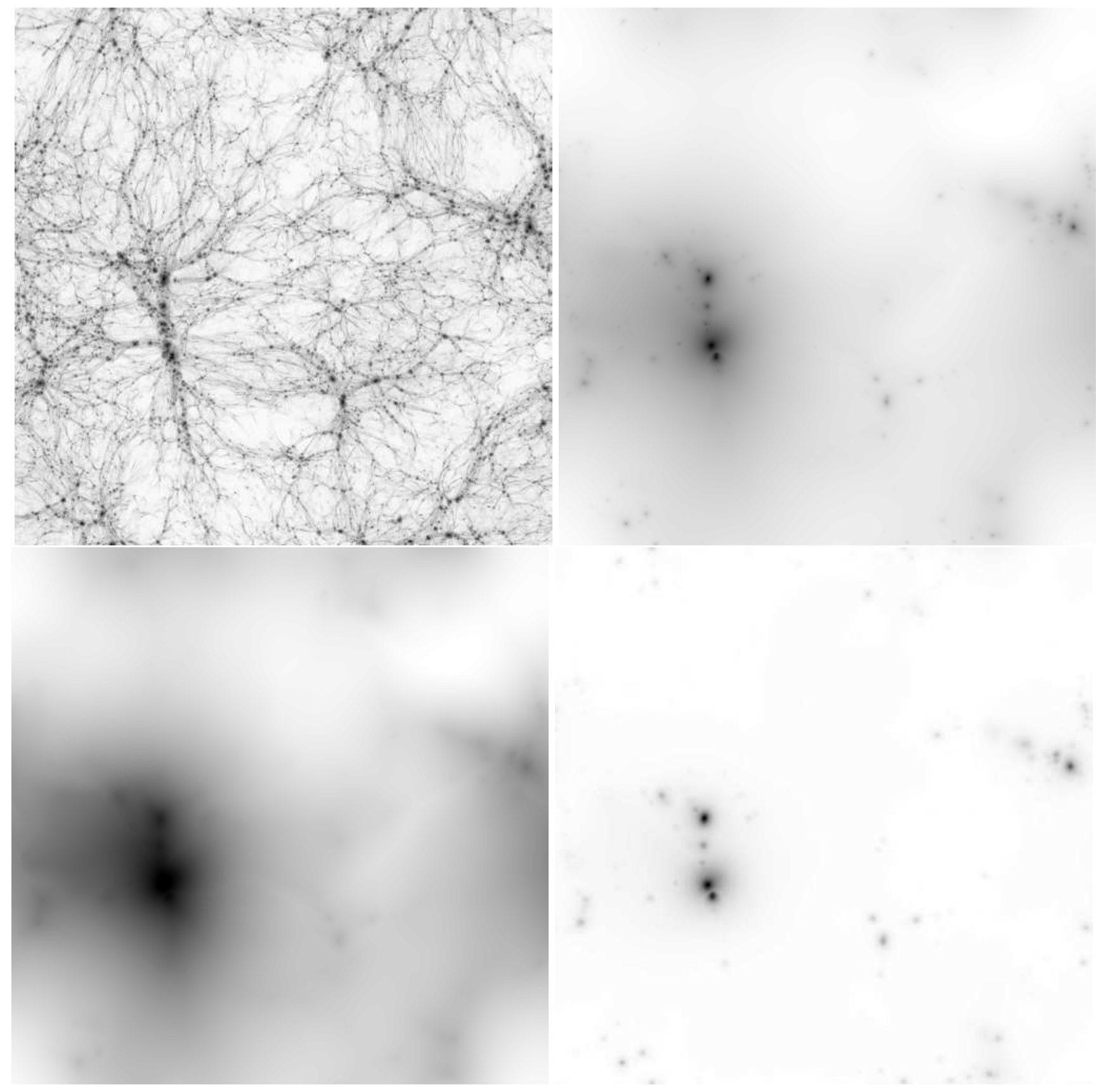

FIG. 5: Slices through a full DGP simulation with $L_{\mathrm{box}}=64 \mathrm{Mpc} / h$ at $z=0$. Top left: density in logarithmic scale; top right: dynamical potential $\Psi$; bottom left: brane-bending mode $\varphi$; bottom right: non-linear suppression of $\varphi$ field: $\varphi_{\mathrm{NL}} \equiv \varphi-\varphi_{L}$ (see text).

\section{RESULTS}

In order to get a visual impression of some of the physics in DGP N-body simulations, we show slices through one simulation box at $z=0\left(L_{\text {box }}=64 \mathrm{Mpc} / h\right)$ in Fig. 5 . The slices are 64 cells thick, and for each pixel we take the maximum absolute values of each quantity over the thickness of the slice, for better visibility. The density field (top left; in logarithmic scale) is difficult to distinguish visually from the QCDM result for the same run, as the effects on the power spectrum are at the $\sim 15 \%$ level (Section VIA). The top right panel in Fig. 5 shows the dynamical potential $\Psi$, exhibiting the potential wells of massive collapsed structures. The brane-bending mode $\varphi$ is shown in the lower left panel. On linear scales, $\varphi \approx \varphi_{L}$ is proportional to the potential $\Psi$ (Section IIC), but evidently does 

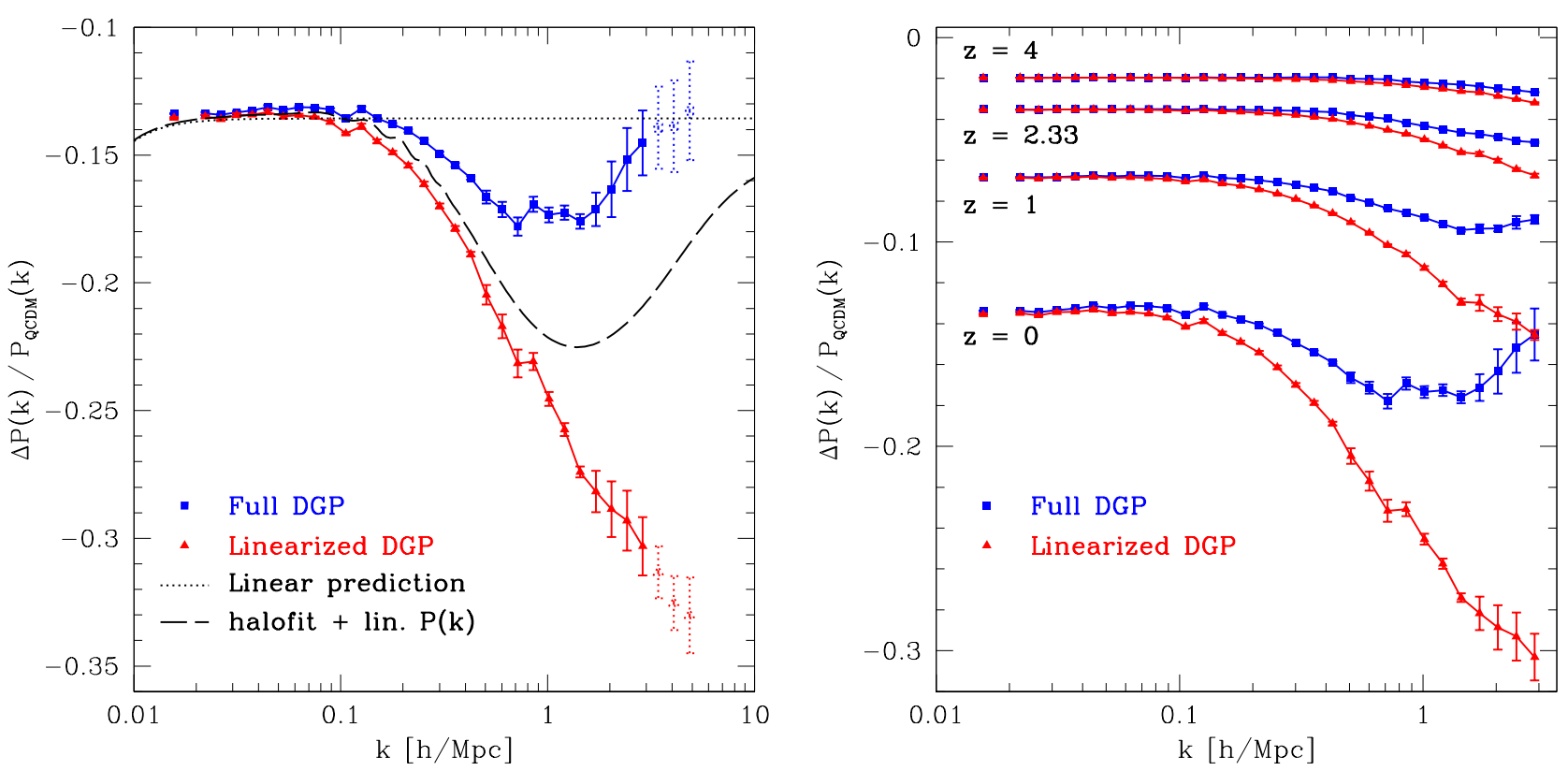

FIG. 6: Left panel: Relative power spectrum deviation of full and linearized DGP simulations from GR (QCDM) at $z=0$. The dashed points at high $k$ extend the range from $k_{\mathrm{Ny}_{\mathrm{y}}} / 8$ to $k_{\mathrm{Ny}} / 4$ and are only intended to be indicative of the trend. The dotted line shows the deviation expected in linear theory, while the dashed line indicates the deviation predicted using linear theory as input for halofit. Right panel: Same as the left panel, but measured in the simulations at different redshifts.

not follow the potential within deeper potential wells, making it appear smoother. To make this more clear, we show the difference of the $\varphi$ solution from the linear value [Eq. (2.10)], $\varphi_{\mathrm{NL}} \equiv \varphi-\varphi_{L}$, in the lower right panel of Fig. 5: $\varphi$ is suppressed $\left(\varphi_{\mathrm{NL}}<0\right)$ in overdense regions, showing the Vainshtein effect at work in a cosmological setting. Note that quite low-mass structures which are not conspicuous in the potential $\Psi$ already lead to a suppression of $\varphi$. See Section VIC for a discussion of the Vainshtein effect in dark matter halos.

\section{A. Power spectrum}

Fig. 6 (left panel) shows the relative deviation of the linearized and full DGP power spectra from the QCDM result at redshift 0 . The error bars are obtained from the 6 separate runs using a bootstrap procedure. By comparing simulation runs with the same initial conditions, and then averaging the deviations, most of the cosmic variance cancels out and we are able to obtain significantly less scatter. Also shown is the predicted deviation in the linear power spectrum. Note first that in the self-accelerated DGP branch, the scalar field mediates a repulsive force, leading to a suppression of the growth of structure. Due to the scale-invariant modification of gravity in linearized quasi-static DGP [Eq. (2.10)], the predicted linear deviation is also scale-independent. On linear scales, $k \lesssim 0.1 h / \mathrm{Mpc}$, both linearized and full DGP simulations agree well with the linear prediction.

Apparently, the full DGP result departs significantly from linearized DGP on quasi-linear to non-linear scales. The Vainshtein effect begins to operate wherever overdensities become of order unity, and suppresses the deviation from GR. Note that small effects of the non-linear $\varphi$ equation can already be seen on quite large scales, $k \sim 0.05 h / \mathrm{Mpc}$ corresponding to $r \sim 60 \mathrm{Mpc} / h$, not far from the acoustic features in the matter power spectrum. While we do not expect dramatic effects on cosmological parameter constraints from BAO, a modeling of these non-linear effects will be necessary for precision constraints in the context of DGP and similar braneworld models.

We also show the deviation of $P_{\mathrm{NL}, \mathrm{DGP}}$ from $P_{\mathrm{NL}, \mathrm{QCDM}}$, where $P_{\mathrm{NL}}$ is calculated from the corresponding linear power spectrum using the standard halofit prescription. halofit describes the linearized DGP power spectrum reasonably well up to $k \lesssim 0.4 \mathrm{~h} / \mathrm{Mpc}$, in agreement with the findings of [52], while it follows neither the linearized nor full DGP at higher $k$. We have not explored whether phenomenological modifications of halofit allow for an improvement of the fit.

The dashed points at high $k$ extend the range in frequency up to $k_{\mathrm{Ny}} / 4$ of our smallest box, $64 \mathrm{Mpc} / h$. While resolution effects are still expected to cancel out to first order in this representation, these points only serve to 


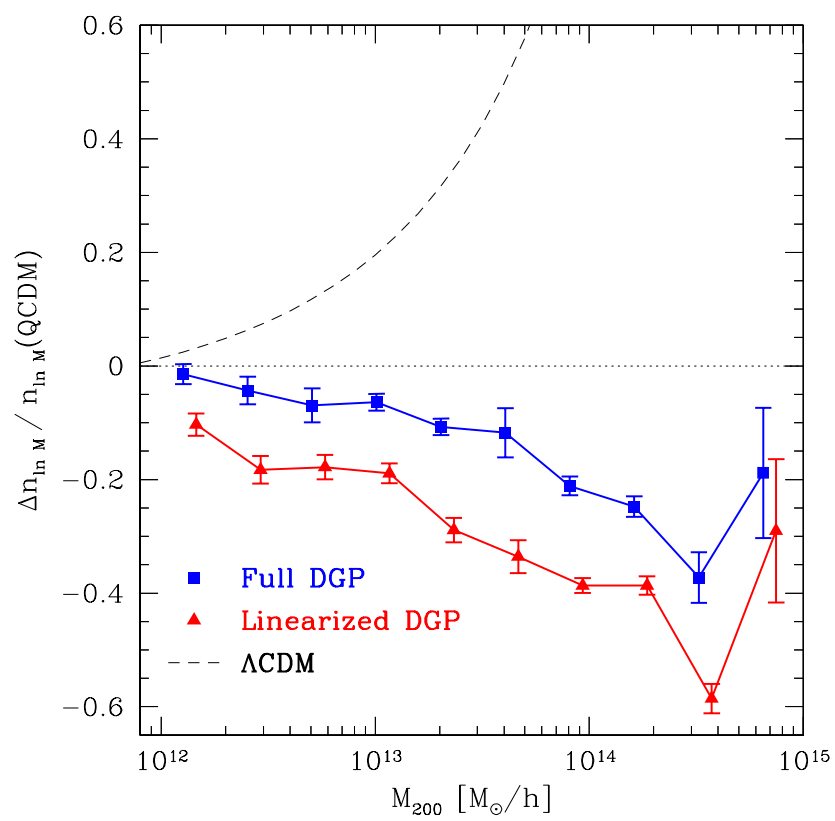

FIG. 7: Relative deviation of the halo mass function $n_{\ln M} \equiv d n / d \ln M_{200}$ measured in full and linearized DGP simulations from GR $(\mathrm{QCDM})$ at $z=0$ (points). The dashed line shows the relative deviation of the predicted $\Lambda$ CDM mass function (for the same $\left.\Omega_{m}, h\right)$ from QCDM (see Fig. 4).

indicate that the trends seen for full and linear DGP at lower $k$ continue towards smaller scales.

The right panel of Fig. 6 shows the evolution of the modified gravity effects on the power spectrum as function of redshift. On large scales $k \lesssim 0.1 \mathrm{~h} / \mathrm{Mpc}$, the deviation is almost scale-free and evolves as predicted by linear theory. At earlier times, the density field is non-linear only on smaller scales. Hence, the Vainshtein effect becomes visible in the power spectrum only at higher $k$. However, it does affect the power spectrum deviation significantly already at $z>1$.

\section{B. Halo mass function}

The abundance of massive dark matter halos is a sensitive probe of the growth of structure in the Universe [5356]. In particular, the number of the most massive halos which host galaxy clusters depends exponentially on the amplitude of the matter power spectrum. Fig. 7 shows the effect of the modification of gravity in DGP on the halo mass function, relative to the QCDM effective dark energy cosmology. We investigated the effect of the smoothing used in the full DGP simulations by comparing linearized DGP simulations with and without smoothing, as done for the power spectrum (Section IV B). Above our rather conservative mass threshold for each box, we only found a small effect of the smoothing on halo masses in the linearized DGP simulations. Since the $\varphi$ field is in any case suppressed within halos due to the Vainshtein mechanism, we expect the smoothing effects to be even smaller for the full DGP simulations.

As expected, the full DGP simulations are somewhat closer to QCDM than the linearized DGP case in Fig. 7. The suppression in the mass function is significant for $M_{200} \gtrsim 10^{13} M_{\odot} / h$, reaching more than $30 \%$ for massive cluster-size halos. The suppression is smaller for lower-mass halos. This is presumably because these halos formed earlier in cosmic history, when the modified gravity effects of DGP were significantly smaller.

Note that when compared to $\Lambda \mathrm{CDM}$, this suppression comes in addition to the larger suppression of the mass function due to the expansion history of DGP (see dashed line in Fig. 7). However, we expect the effect of the DGP modification of gravity to be fairly independent of the expansion history. In particular, we expect a similar effect on the halo mass function in generalized braneworld models which exhibit an expansion history close to $\Lambda$ CDM [32, 38]. Hence, the mass function is expected to be a sensitive probe for braneworld modified gravity models, as is the case for $f(R)$ gravity [20]. Note that for the normal branch of braneworld gravity, the sign of the effect in Fig. 7 will be reversed, leading to an enhancement of the number of massive halos. 

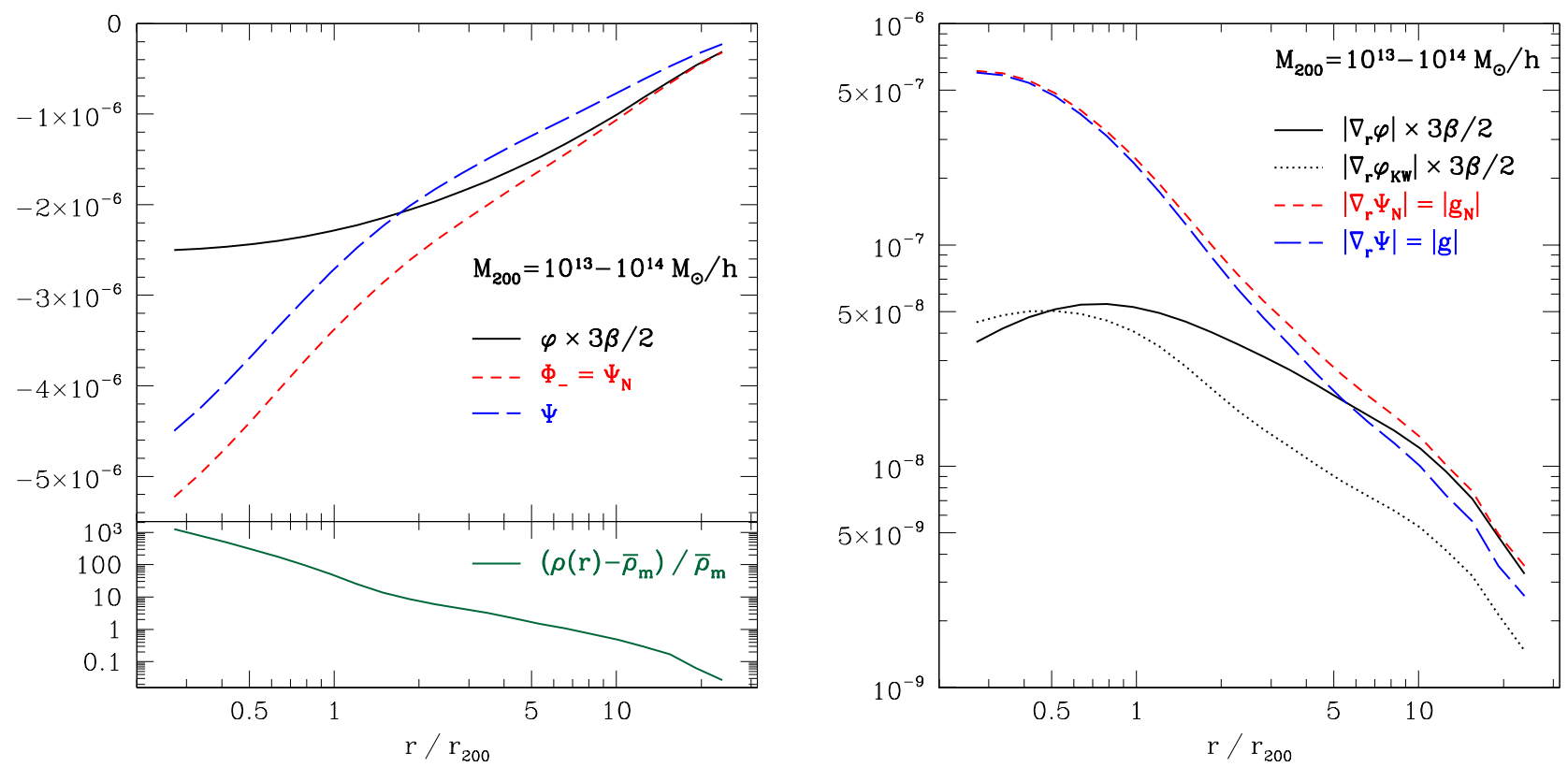

FIG. 8: Left panel: Average halo profiles of $\varphi$, the dynamical potential $\Psi$, and the lensing potential $\Phi_{-}=\Psi_{N}$ in the DGP simulations at $z=0$, for halos between $10^{13}$ and $10^{14} M_{\odot} / h$. The lower panel shows the average profile of the matter overdensity $\delta$ for the same halos. Right panel: Average halo profiles of the radial gradient of $\varphi$, the DGP potential $\Psi$, and Newtonian potential $\Psi_{N}$, showing that $g_{r}=-\nabla_{r} \Psi \rightarrow g_{N}=-\nabla_{r} \Psi_{N}$ in the inner regions of halos, i.e. GR is restored. Also shown is the brane bending mode calculated in the approach of [38], $\varphi_{\mathrm{KW}}$ (see text), for the same halos as in the left panel.

\section{Brane bending mode and Vainshtein effect}

Fig. 8 (left panel) shows the average profiles of the brane-bending mode $\varphi$, the dynamical potential $\Psi$, and the lensing potential $\Phi_{-}$of dark matter halos in the full DGP simulations. The brane-bending mode has been scaled by $3 \beta / 2$, so that it agrees with $\Phi_{-}=\Psi_{N}$ for the linearized solution. We only use our highest resolution simulation box for this measurement $\left(L_{\mathrm{box}}=64 \mathrm{Mpc} / h\right)$. The inner radius of the profiles is given by one grid cell. The profiles were measured by spherically averaging many lines of sight for each halo with $10^{13} M_{\odot} / h<M_{200}<10^{14} M_{\odot} / h$, and then stacked, scaling each profile to the radius $r_{200}$ of the respective halo. This was done in order to reduce the significant scatter in the profiles.

While the lensing potential $\Phi_{-}$obeys the standard Poisson equation in DGP, the dynamical potential $\Psi$ receives a contribution from the brane bending mode [Eq. (2.8)]. Apparently, the $\varphi$ field flattens out towards the halo center, which is in qualitative agreement with the non-linear field solution for a spherical mass (see, e.g. Fig. 1). Note that $\varphi$ turns away from the linear solution when the overdensity is of order a few, in agreement with the estimate in Section II C.

The quantity which is actually observable however is the gradient of the field, shown in Fig. 8 (right panel), which gives essentially the deviation of the acceleration from the Newtonian acceleration. For each halo, we measure the radial gradient relative to the halo center. We again scaled $\nabla_{r} \varphi$ by $3 \beta / 2$ to match the linearized solution to $\nabla_{r} \Psi_{N}$. From the halo exterior towards the interior, the gradient of $\varphi$ grows initially, turns around at $r \sim 0.8 r_{200}$, and shrinks for even smaller radii. As is clearly shown by comparing the dynamical potential $\Psi$ with its Newtonian value, $\Psi_{N}$, any observable deviations from GR are indeed suppressed within massive halos. We find that for halos between $10^{12}$ and $10^{13} M_{\odot} / h$, the deviation in the acceleration is around $\Delta g / g_{N} \approx 0.2 /(3 \beta)$ in the inner parts, while it is suppressed down to $\Delta g / g_{N} \approx 0.05 /(3 \beta)$ for halos around $10^{14} M_{\odot} / h$. This trend with mass is expected, given that $r_{*} \propto M^{1 / 3}$ for the spherically symmetric solution.

In order to model the behavior of the brane bending mode in the cosmological context, we can make use of the two limiting cases presented in Section II. One is the spherically symmetric solution (Section IID), which can be used in modeling a spherical collapse of dark matter halos in DGP [57]. Moreover, Khoury and Wyman [38] have based the approximate field solution in their N-body simulation on this limiting case. More realistically however, cosmic structure forms by collapsing into non-radially symmetric structures such as filaments. The plane wave density perturbation can serve as another, albeit rather extreme, test case. For such a perturbation, the non-linear self-coupling of $\varphi$ 
vanishes (Section IIE). Hence, we expect the Vainshtein effect to be typically weakened in a non-radially symmetric setting, compared to the spherically symmetric case.

In their ansatz, Khoury and Wyman assumed that the spherically symmetric solution Eqs. (2.14)-(2.15) holds wherever the field becomes non-linear. Since in this solution, the gravitational constant is rescaled locally by $1+\Delta(r)$, the Poisson equation for $\Psi$ can be written in this ansatz as:

$$
\nabla^{2} \Psi=4 \pi G[1+\Delta(\delta)] a^{2} \bar{\rho}_{m} \delta
$$

where $\Delta$ is now a function of the local overdensity. Substituting $\delta \sim M / \bar{\rho}_{m} r^{3}$ in Eq. (2.16) [or Eq. (2.12)] we see again that $\left(r_{*} / r\right)^{3} \sim\left(H_{0} r_{c}\right)^{2} \beta^{-2} \Omega_{m} \delta$, with an order unity coefficient. Khoury and Wyman [38] chose:

$$
\left(\frac{r_{*}}{r}\right)^{3}=\varepsilon=\frac{8 H_{0}^{2} r_{c}^{2}}{9 \beta^{2}} \Omega_{m} \delta \Rightarrow \Delta(\delta)=\frac{2}{3 \beta}(\sqrt{1+\varepsilon}-1) / \varepsilon
$$

Hence, in the DGP model we simulated, $\varepsilon \approx 0.3 \delta$. Given the density field in our DGP simulations, we can solve Eq. (6.1) by Fourier transform as done in [38], subtract the Newtonian potential, and compare the brane bending mode $\varphi_{\mathrm{KW}}$ from this ansatz with our numerical solution of the full $\varphi$ equation.

Fig. 8 (right panel) shows the result for stacked halo profiles of the radial gradient of $\varphi_{\mathrm{KW}}$. While $\varphi_{\mathrm{KW}}$ shows a qualitatively similar behavior to the full solution, the suppression due to the Vainshtein effect appears to set in at significantly larger radii in $\varphi_{\mathrm{KW}}$ than in the full solution. This is qualitatively in agreement with our expectation that non-radially symmetric configurations experience a weaker non-linear suppression. The fact that $\varphi_{\mathrm{KW}}$ does not approach the linear solution at large radii is a result of the approximation Eq. (6.1), as was derived in the appendix of [38]. The precise tensorial structure of Eq. (2.6) restores $\varphi$ to the linear solution at large distances $r \gg r_{V}$. It would be worth investigating whether this simplified (and computationally much less expensive) approach can be extended to recover the linear $\varphi$ solution at large scales, an essential feature of the full brane-bending mode interactions.

Although we did not compare our results with a full simulation based on the spherically symmetric approach of [38], these results seem to indicate that this approximation overestimates the non-linear suppression of the brane-bending mode, which might affect observables such as the power spectrum or halo mass function. We point out however that the crossover scale $r_{c}$ in the braneworld-inspired model considered in [38] is an order of magnitude smaller than our $r_{c}$, and the non-linearity in Eqs. (6.1)-(6.2) only becomes effective at much higher overdensities $\delta=O(100)$, so that for the models studied in [38], the solution is possibly less sensitive to this approximation.

\section{CONSTRAINTS ON THE SELF-ACCELERATING DGP MODEL}

We now briefly discuss the impact of our simulation results on cosmological constraints on the self-accelerating DGP model without $\Lambda$. First, regarding, baryon acoustic oscillations, our results for the DGP power spectrum on quasi-linear scales show that non-linear effects are not significantly enhanced at the BAO scale. We estimate that any modified gravity corrections to the BAO scale are below the percent level, and hence well within the uncertainties of current BAO measurements [58, 59]. Including baryon acoustic oscillations will increase the power of the combined CMB and Supernova constraints on the self-accelerating DGP model [30] with non-zero curvature to $4-5 \sigma$ [60].

Second, weak lensing measurements constrain the amplitude of the matter power spectrum today, which in this model is significantly smaller due to the suppression of growth by the earlier onset of acceleration and the repulsive force mediated by the brane-bending mode. The linear power spectrum normalization at $z=0$ in the models we simulated is $\sigma_{8}(\mathrm{QCDM})=0.56$ and $\sigma_{8}(\mathrm{DGP})=0.52$, while for a $\Lambda$ CDM model with the same primordial normalization, it is $\sigma_{8}(\Lambda \mathrm{CDM})=0.66$. We found that the non-linear matter power spectrum in the full DGP simulations is always below that of QCDM (up to $k \lesssim 6 h / \mathrm{Mpc}$, Fig. 6). Hence, the suppression of the non-linear power spectrum in the self-accelerating DGP model corresponds to a reduction in the inferred linear normalization $\sigma_{8}$ today of at least 0.1 , with respect to a $\Lambda$ CDM model with the same initial conditions. Such a deviation is disfavored by about 1.5 standard deviations by current weak lensing measurements [61].

The abundance of massive dark matter halos is probed by cluster surveys. We showed in Section VIB that the abundance of halos above $10^{14} M_{\odot} / h$ is suppressed by around $20 \%$ relative to QCDM, which itself only predicts half as many halos in that mass range as a $\Lambda$ CDM model with the same primordial power. The latter again corresponds to a shift in $\sigma_{8}$ by 0.1 , which is constrained to more than $4 \sigma$ by X-ray cluster measurements [56], when taking into account the systematic errors. 


\section{CONCLUSIONS}

The N-body simulations of DGP gravity we presented here show how the self-interactions of the brane bending mode $\varphi$, which are responsible for restoring General Relativity in dense environments, influence the formation of structure in the universe. In the self-accelerating DGP model we simulated, this scalar field mediates a repulsive force, effectively weakening gravity. We indeed find that the field solution turns away from the linearized solution whenever the matter overdensity $\delta$ becomes of order a few, and that the repulsive force is suppressed by more than an order of magnitude compared to its linearized value in the center of massive halos. We also compared our full solution to that obtained in the approximate ansatz of [38]. While their ansatz agrees well with our results in the densest regions, it seems to overestimate the non-linear suppression in less dense regions, such as the outer regions and environments of halos. This is in line with our finding that the non-linear suppression of $\varphi$ is weaker for non-spherically symmetric configurations.

The non-linear matter power spectrum in the DGP simulations shows that the suppression due to the repulsive $\varphi$ field is amplified on non-linear scales for the linearized DGP simulations. In the full DGP simulations, this suppression is smaller, and eventually turns around on Mpc scales. The deviations between linerized and full DGP power spectra are noticeable already on quasi-linear scales, $k \gtrsim 0.1 \mathrm{~h} / \mathrm{Mpc}$. At the BAO scale, modified gravity effects on the power spectrum are at the percent-level.

We found that the abundance of massive dark matter halos is significantly suppressed in the DGP simulations, compared to a standard gravity simulation with the same expansion history. Again, the non-linear suppression of the $\varphi$ field alleviates this suppression, and hence has to be taken into account when using observations to constrain

this type of modified gravity. The effect on the halo mass function is in fact large enough to make this an interesting observational probe of more general braneworld scenarios.

Independently of the CMB constraints [30], our results on the non-linear structure formation strongly constrain the self-accelerating DGP model (without $\Lambda$ ). In the future, we plan to extend our simulations to the normal branch of DGP, and more general braneworld(-inspired) models [32, 34, 38]. The key part of the simulations, solving for the non-linear interactions of the brane-bending mode, is generic to all of these models, and hence the code will be readily generalized to these cases. We also plan to attempt a modeling of the modified gravity effects, in the context of the halo model for example. This model can then serve as a framework for cosmological constraints on braneworld gravity which take into account the non-linear mechanisms inherent to this model consistently. Simulations of both $f(R)$ gravity and DGP have shown that most interesting phenomena appear on scales of 1 to tens of Mpc, and in the abundance and environments of clusters. Fortunately, these scales are well accessible to observations, e.g., through weak lensing, the Lyman- $\alpha$ forest, and cluster surveys, which can then be used as precision probes of gravity on cosmological scales.

\section{Acknowledgments}

I am indebted to Scott Dodelson, Wayne $\mathrm{Hu}$, and Andrey Kravtsov for invaluable input and guidance. I would like to thank Hiro Oyaizu and Marcos Lima for our previous collaborative work on the code and analysis, and Angela Olinto, Mike Gladders, Cora Dvorkin, Sam Leitner, Michael Mortonson, and Amol Upadhye for discussions. The support of the Fermilab computing staff is gratefully acknowledged.

The simulations used in this work have been performed on the Joint Fermilab - KICP Supercomputing Cluster, supported by grants from Fermilab, Kavli Institute for Cosmological Physics, and the University of Chicago. This work was supported by the Kavli Institute for Cosmological Physics at the University of Chicago through grants NSF PHY-0114422 and NSF PHY-0551142.

\section{APPENDIX A: CODE IMPLEMENTATION AND DISCRETIZATION}

This appendix describes details of the N-body code implementation, focusing on the relaxation solver for Eq. (2.6) as the main non-standard part of the code. The code is written in $\mathrm{C}++$, uses OpenMP for parallelization of most time-critical operations, and employs FFTW [62] for the Fast Fourier Transforms. 


\section{Code units}

The comoving code units follow the convention used in $[63,64]$, where

$$
\tilde{\mathbf{x}}=a^{-1} \frac{\mathbf{x}}{r_{0}}, \quad \tilde{t}=\frac{t}{t_{0}}, \quad \tilde{\mathbf{p}}=a \frac{\mathbf{v}}{v_{0}}, \quad \tilde{\Psi}=\frac{\Psi}{\psi_{0}}, \quad \tilde{\rho}=a^{3} \frac{\rho}{\rho_{m 0}},
$$

and

$$
r_{0}=\frac{L_{\mathrm{box}}}{N_{g}}, \quad t_{0}=H_{0}^{-1}, \quad v_{0}=\frac{r_{0}}{t_{0}}, \quad \rho_{m 0}=\Omega_{m} \rho_{\text {crit }, 0}, \quad \psi_{0}=v_{0}^{2} .
$$

In the above definitions, $L_{\text {box }}$ is the comoving simulation box size in $\mathrm{Mpc} / h, N_{g}$ is the number of grid cells in each direction, $H_{0}$ is the Hubble parameter today, $\rho_{\text {crit }, 0}$ is the critical density today, and $\Omega_{m}$ is the fraction of non-relativistic matter today relative to the critical density.

Transformed to code units, the equation for $\varphi$ becomes:

$$
L(\varphi) \equiv \tilde{\nabla}^{2} \varphi+\frac{{\tilde{r_{c}}}^{2}}{3 \beta a^{2}}\left[\left(\tilde{\nabla}^{2} \varphi\right)^{2}-\left(\tilde{\nabla}_{i} \tilde{\nabla}_{j} \varphi\right)^{2}\right]=f \equiv \frac{\Omega_{m}}{a \tilde{c}^{2} \beta} \tilde{\delta} .
$$

Here, $\tilde{\nabla}$ acts with respect to code coordinates, $\tilde{r_{c}}=r_{c} / r_{0}, \tilde{c}=1 / v_{0}$, and $\tilde{\delta}=\delta \rho / \overline{\rho_{m}}$ is the matter overdensity on the grid, determined from the particle positions. Note that we have not yet scaled $\varphi$ to $\psi_{0}$. After solving Eq. (A3), $\varphi$ is added to the standard Newtonian potential, which is solved for using a Fast Fourier Transform, to obtain the dynamical potential in DGP:

$$
\tilde{\Psi}=\tilde{\Psi}_{N}+\frac{1}{2} \frac{\varphi}{\psi_{0}}
$$

\section{Discretization of $\varphi$ equation}

We employ standard second-order symmetric differences for the discretization of the second derivatives in Eq. (A3):

$$
\begin{aligned}
\tilde{\nabla}_{x} \tilde{\nabla}_{x} \varphi_{i, j, k} & =\frac{1}{h^{2}}\left(\varphi_{i+1, j, k}+\varphi_{i-1, j, k}-2 \varphi_{i, j, k}\right), \\
\tilde{\nabla}_{x} \tilde{\nabla}_{y} \varphi_{i, j, k} & =\frac{1}{4 h^{2}}\left(\varphi_{i+1, j+1, k}-\varphi_{i+1, j-1, k}-\varphi_{i-1, j+1, k}+\varphi_{i-1, j-1, k}\right),
\end{aligned}
$$

and correspondingly for derivatives with respect to $y, z$. Here, $i, j, k$ stand for the grid indices, and the step size $h=1$ for the base grid and $h=2^{n}$ for the grid of refinement level $n$. The finite differences are evaluated before squaring in calculating the non-linear terms in Eq. (A3).

We have tried different discretizations, such as going to higher order in the finite differences, and solving for the deviation of $\varphi$ from the solution of the linearized equation [Eq. (2.10)], instead of solving for $\varphi$ itself. The performance of those discretizations is comparable to or worse than the simple discretization Eqs. (A5)-(A6). This is understandable, since going to higher order amounts to making the derivative operations less local in order to be sensitive to error modes of longer wavelength. However, in our multigrid relaxation scheme, this is already done efficiently by the coarser grids, so going to higher order in a single relaxation does not improve performance. Hence, we stay with the straightforward discretization, Eqs. (A5)-(A6).

\section{Relaxation algorithm}

In general, a relaxation scheme operates by iteratively obtaining a better approximation to the solution $\varphi^{(i+1)}$ given a previous guess, $\varphi^{(i)}$. On the grid, the solution is updated successively for each cell $(i, j, k)$ :

$$
\varphi_{i, j, k}^{(i+1)} \leftarrow F\left(\varphi_{l, m, n}^{(i)}\right),
$$

where $F$ is the discretized field equation solved for $\varphi_{i, j, k}$. In case of a linear field equation, $F$ can be solved for $\varphi_{i, j, k}$ straightforwardly. However, in our case the field equation is non-linear in $\varphi$, and we instead solve for $F$ iteratively via Newton's method. Writing the field equation Eq. (A3) as $L(\varphi)=f$, we need to solve:

$$
L\left(\ldots, \varphi_{i, j, k}, \ldots\right)-f_{i, j, k}=0
$$


for $\varphi_{i, j, k}$, where we have suppressed the dependences of $L$ on neighboring grid cells. By expanding $L\left(\varphi_{i, j, k}\right)$ in a Taylor series around the current approximation, one step of Newton's method works by updating $\varphi_{i, j, k}^{(i)}$ with:

$$
\varphi_{i, j, k}^{(i+1)} \leftarrow \varphi_{i, j, k}^{(i)}-\frac{L\left(\ldots, \varphi_{i, j, k}^{(i)}, \ldots\right)-f_{i, j, k}}{\partial L / \partial \varphi_{i, j, k}^{(i)}} .
$$

Our relaxation step is thus given by Eq. (A9), where $\partial L / \partial \varphi_{i, j, k}$ is determined from Eq. (A3) and the discretization Eqs. (A5)-(A6). In practice, the order in which we loop over grid cells in performing the relaxation Eq. (A9) is important, since each relaxation step depends on the neighboring grid cells. In particular, we need to take care when parallelizing the relaxation. We use a generalized red-black scheme (see, e.g., [42, 47]), by successively running over cells with $(i+j+k)$ modulo $4=n, n=0,1,2,3$. This was done in order to break dependences due to neighboring cells within each set, in particular due to the mixed derivatives Eq. (A6), so that each set can be efficiently parallelized. We experimented with different ordering schemes and found that this choice performed best.

Now, assume we have an approximate solution $\varphi$ to the field equation Eq. (A3) on the fine grid $\Omega^{h}$, which differs from the true solution $\bar{\varphi}$ by the error $e, \bar{\varphi}=\varphi-e$. Further, the residual is defined as $r=L(\varphi)-f$. Since $L(\bar{\varphi})=f$ by assumption, we obtain the following residual equation:

$$
L(\varphi)-L(\varphi-e)=r
$$

We expect that the relaxation on the fine grid has removed most small-scale error modes, so $e$ mainly consists of longer wavelength modes. Thus, we will solve for $e$ on the coarser grid $\Omega^{2 h}$, and then correct the approximate solution $\varphi$ for this error. On the coarse grid $\Omega^{2 h}$, Eq. (A10) reads:

$$
L^{2 h}\left(I_{h}^{2 h} \varphi^{h}\right)-L^{2 h}\left(I_{h}^{2 h} \varphi^{h}-e^{2 h}\right)=I_{h}^{2 h}\left(L^{h}\left(\varphi^{h}\right)-f^{h}\right) .
$$

Here, the superscripts denote which grid a given quantity is defined on, and $I_{h}^{2 h}$ is the restriction operator mapping fields from the fine grid to the coarse grid. Note that Eq. (A11) is of the same form as the $\varphi$ equation on the coarse grid, $L^{2 h}\left(\varphi^{2 h}\right)=f^{2 h}$, except with a different right-hand side. Hence, Eq. (A11) is solved for $e^{2 h}$ using the same algorithm as the original equation, but at one grid level higher. Once Eq. (A11) is solved, we can correct $\varphi^{h}$ for the error:

$$
\varphi^{h} \leftarrow \varphi^{h}-I_{2 h}^{h} e^{2 h},
$$

where $I_{2 h}^{h}$ is the interpolation operator mapping the error from the coarse grid to the fine grid.

In summary, the multigrid relaxation proceeds as follows:

- Relax Eq. (A3) on the fine grid $\Omega^{h} N_{I}$ times. Compute the residual $r^{h}=L^{h}\left(\varphi^{h}\right)-f^{h}$.

- Solve Eq. (A11) for the error $e^{2 h}$ :

- Relax Eq. (A11) on $\Omega^{2 h} N_{I}$ times. Compute the residual (l.h.s.- -r.h.s.) of Eq. (A11).

- Solve Eq. (A11) for $e^{4 h}$ :

$\ldots$

- Correct $e^{2 h}$ using $I_{4 h}^{2 h} e^{4 h}[$ Eq. (A12)].

- Correct $\varphi^{h}$ using $I_{2 h}^{h} e^{2 h}[$ Eq. (A12)].

Thus, the relaxation proceeds as a nested loop going down to the coarsest grid, and then correcting the solutions on the successively finer grids. One such iteration through all grid levels is called a $V$-cycle. The coarsest grid we use has 4 cells on a side, which corresponds to a refinement of $2^{7}$ for a $N_{g}=512$ base grid.

For the interpolation operator $\Omega^{2 h} \rightarrow \Omega^{h}$, we use standard bilinear interpolation (consistent with the cloud-in-cell scheme used for assigning densities and measuring accelerations on the grid). For the restriction $\Omega^{h} \rightarrow \Omega^{2 h}$, we use full-weighting, the transpose of the bilinear interpolation operator. See the appendix in [42] for an explicit definition.

In order to ensure convergence at all times, we adopt a large value of $N_{I}=10$, at the expense of computing time. Usually, one V-cycle reduces the residual $\mathcal{L}=\sqrt{\left\langle r^{2}\right\rangle}$ by 1-2 orders of magnitude. We stop the relaxation when either $\mathcal{L}<10^{-14}$ is reached, or the convergence stalls at a certain level of $\mathcal{L}$. Either of this usually happens within $4 \mathrm{~V}$-cycles. The value of $\mathcal{L}$ for each time step is logged, allowing for a monitoring of the convergence status in the simulations. 

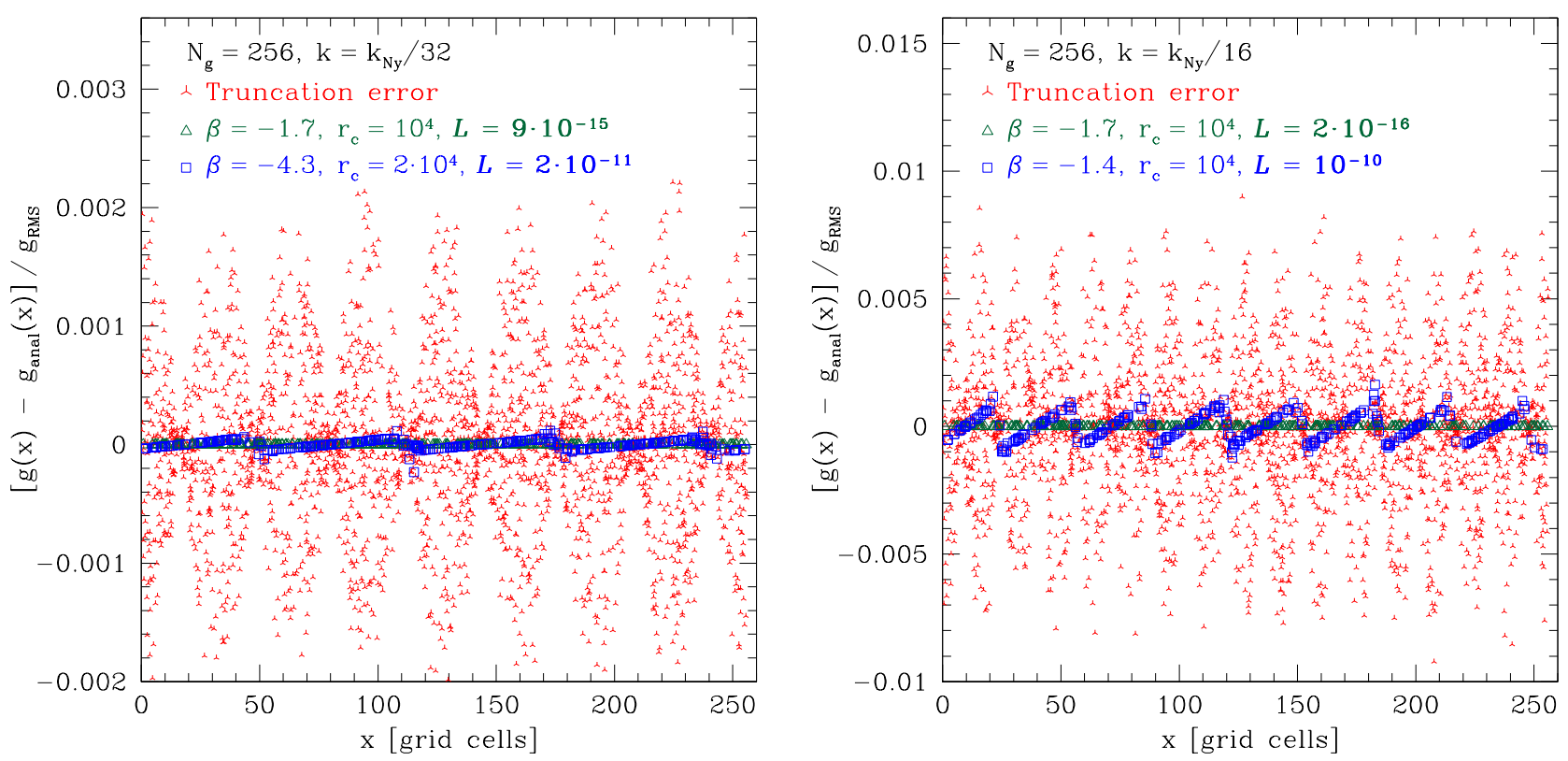

FIG. 9: Truncation errors (red points) and residual errors in the $\varphi$ field solution (green, blue points) for sine waves with $k=k_{\mathrm{Ny}} / 32$ (left panel) and $k=k_{\mathrm{Ny}} / 16$ (right panel). The parameters used for the field equation are shown (with $r_{c}$ in $\mathrm{Mpc} / h)$, as well as the RMS residuals $\mathcal{L}$ of the final solution. The errors are shown as deviations of the acceleration measured at random points from the expectation, scaled to the RMS acceleration of the sine wave (see text).

\section{APPENDIX B: SINE WAVE TEST}

In this section, we use the fact that the full $\varphi$ solution is identical to the linearized solution for a plane wave perturbation (Section IIE) to test our code for perturbations on different length scales. We consider a simple sine wave density perturbation, with $k$-vector chosen to lie along the $x$-axis:

$$
\delta(x)=\frac{\delta \rho}{\rho}=A \sin k x .
$$

In this case, the solution to the non-linear $\varphi$ equation is identical to the linear solution:

$$
\varphi(x)=\frac{2}{3 \beta} \Psi_{N}(x)=\frac{2}{3 \beta} \frac{3 \Omega_{m}}{2 a k^{2}} A \sin k x .
$$

Hence, the exact acceleration is given by:

$$
g(x)=\left(1+\frac{1}{3 \beta}\right) g_{N}(x)=\left(1+\frac{1}{3 \beta}\right) \frac{3 \Omega_{m}}{2 a k} A \cos k x .
$$

For a given level of residuals $\mathcal{L}$, we now demand that the errors due to the approximate solution of the $\varphi$ equation of motion are small compared to the unavoidable truncation errors which are encurred by taking the gradient of the potential on the grid.

In the following, we set $a=1, \Omega_{m}=0.3$, and the number of grid cells is set to $256^{3}$. The truncation errors are measured by assigning the analytical solution for the potential to each grid point, and comparing the exact acceleration to the one obtained by derivation and interpolation from the grid. The truncation errors for two sine waves of different wavelength are shown as deviations in the acceleration in Fig. 9 (red points). We scale the deviations to the RMS acceleration of the sine wave, $g_{\mathrm{RMS}}=3 \Omega_{m} /(2 \sqrt{2} a k) A$, in order to avoid far outliers obtained near the zeros of the sine wave when scaling to the exact solution at each point. For shorter wavelength modes, the truncation error increases and reaches order unity for waves on the Nyquist scale, as expected.

In order to estimate the additional error in the acceleration made due to insufficient convergence of the solution, we compare the acceleration measured from the non-linear field solution coming out of the relaxation solver, to the 


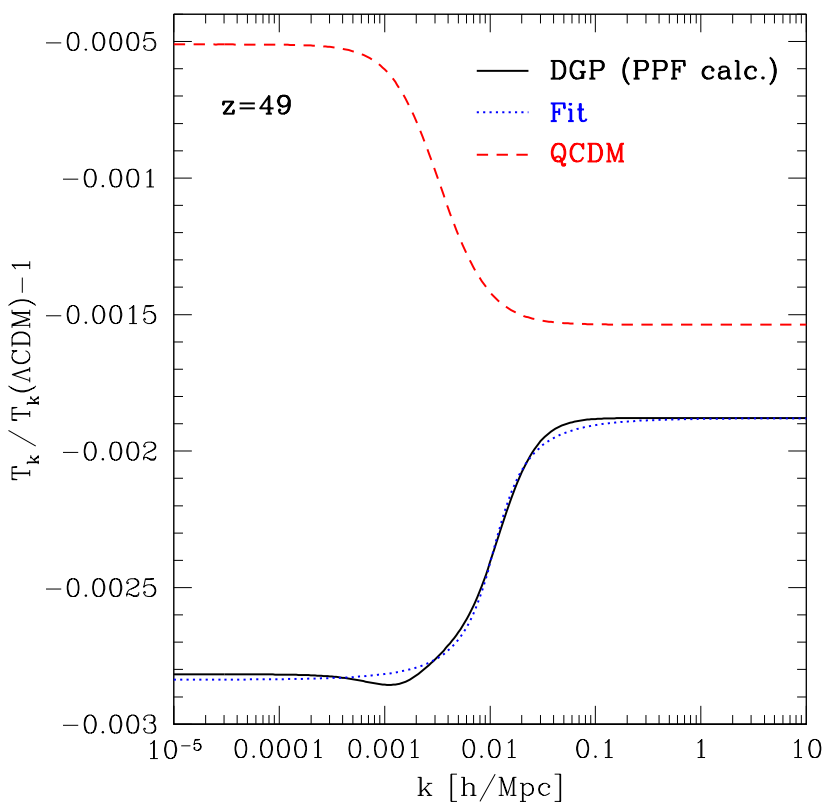

FIG. 10: Deviation of the DGP and QCDM matter transfer functions from $\Lambda$ CDM at the initial redshift of the simulations, $z_{i}=49$. The solid line shows the PPF calculation for DGP, while the blue dotted line is a simple fit used to correct the initial conditions (see text).

corresponding solution of the linearized $\varphi$ equation Eq. (2.10). We vary the parameters $\beta$ and $r_{c}$ which control the strength of the non-linearity in Eq. (2.6). In Fig. 9, we show the measured deviations in the acceleration from the linear solution for different values of $\beta$ and $r_{c}$, and in particular for the largest values that reached convergence. The final residuals of the $\varphi$ solution in each case are also shown.

Apparently, the errors in the acceleration are completely negligible for residuals $\mathcal{L} \leq 10^{-14}$, consistent with results we found for other test cases such as the spherical mass. For sufficiently strong non-linearity and short wavelengths, residuals of order $\mathcal{L} \sim 10^{-11}-10^{-10}$ are reached which lead to measurable deviations in the acceleration. However, for the residual values up to $\sim 10^{-10}$ that we explored, the error due to the incomplete convergence is very small compared to the truncation error in all cases. Hence, we conclude that for residuals smaller than the conservative bound of $10^{-10}$, the $\varphi$ solution we obtain is sufficiently accurate in order not to bias the particle dynamics.

\section{APPENDIX C: CORRECTING INITIAL CONDITIONS FOR DGP}

Due to the additional term $H / r_{c}$ in the Friedmann equation, the self-accelerating DGP model starts to deviate from a $\Lambda \mathrm{CDM}$ expansion history at relatively high redshifts. In addition, there are modifications to the growth of horizon-scale modes. For this reason, even at the initial redshift of our simulations, $z_{i}=49$, there are small departures in the matter transfer function in DGP as compared to a $\Lambda$ CDM model with the same early-Universe parameters $\Omega_{m}=1-\Omega_{\Lambda}, \Omega_{b}, h, n_{s}, A_{s}$.

In order to take these differences into account, we calculate the dark matter transfer function $T(k)$ at $z_{i}$ for DGP and QCDM, using the PPF approach described in [30,65], and for the corresponding flat $\Lambda$ CDM model. We do not include the effects of radiation on the transfer function, which is not necessary since we are only interested in the relative deviation of the DGP $T(k)$ from $\Lambda$ CDM. Fig. 10 shows the relative deviation in $T\left(k, z_{i}\right)$ from $\Lambda$ CDM for DGP and QCDM. Both QCDM and DGP transfer functions are slightly suppressed on all scales due to the effects of the earlier onset of acceleration. On super-horizon scales, the transfer function is further suppressed in DGP caused by the transition to $5 \mathrm{D}$ gravity on very large scales. In contrast, $T(k)$ is less suppressed in QCDM because of the fluctuations in the effective dark energy.

We correct the transfer function obtained from CAMB for these small differences at $z_{i}$ by multiplying $T_{\mathrm{CAMB}}(k)$ with $1+f(k)$, where $f(k)$ is a simple arctan +const. function fit to the DGP curve shown in Fig. 10. Hence, these initial conditions are not quite correct for the QCDM simulations. However, the differences are very small on the 
scales probed by our simulations $(k \gtrsim 0.01 \mathrm{~h} / \mathrm{Mpc})$.

[1] A. G. Riess, L.-G. Strolger, S. Casertano, H. C. Ferguson, B. Mobasher, B. Gold, P. J. Challis, A. V. Filippenko, S. Jha, W. Li, et al., Astrophys. J. 659, 98 (2007), arXiv:astro-ph/0611572.

[2] M. Kowalski, D. Rubin, G. Aldering, R. J. Agostinho, A. Amadon, R. Amanullah, C. Balland, K. Barbary, G. Blanc, P. J. Challis, et al., ArXiv e-prints 804 (2008), 0804.4142.

[3] J. Dunkley, E. Komatsu, M. R. Nolta, D. N. Spergel, D. Larson, G. Hinshaw, L. Page, C. L. Bennett, B. Gold, N. Jarosik, et al., ArXiv e-prints 803 (2008), 0803.0586.

[4] T. Giannantonio, R. G. Crittenden, R. C. Nichol, R. Scranton, G. T. Richards, A. D. Myers, R. J. Brunner, A. G. Gray, A. J. Connolly, and D. P. Schneider, Phys. Rev. D 74, 063520 (2006), arXiv:astro-ph/0607572.

[5] D. Pietrobon, A. Balbi, and D. Marinucci, Phys. Rev. D 74, 043524 (2006), arXiv:astro-ph/0606475.

[6] J. A. Frieman, M. S. Turner, and D. Huterer, Ann. Rev. Astron. Astroph. 46, 385 (2008), 0803.0982.

[7] C. M. Will, Living Reviews in Relativity 9, 3 (2006), arXiv:gr-qc/0510072.

[8] P. Zhang, M. Liguori, R. Bean, and S. Dodelson (2007), arXiv:0704.1932 [astro-ph].

[9] B. Jain and P. Zhang, ArXiv e-prints 709 (2007), 0709.2375.

[10] Y.-S. Song and K. Koyama, ArXiv e-prints 802 (2008), 0802.3897.

[11] L. Knox, Y.-S. Song, and J. A. Tyson, Phys. Rev. D 74, 023512 (2006).

[12] F. Schmidt, Phys. Rev. D 78, 043002 (2008), 0805.4812.

[13] Y.-S. Song, Phys. Rev. D 71, 024026 (2005), arXiv:astro-ph/0407489.

[14] Y.-S. Song, ArXiv Astrophysics e-prints (2006), astro-ph/0602598.

[15] S. Tsujikawa and T. Tatekawa, ArXiv e-prints 804 (2008), 0804.4343.

[16] P. Zhang, Phys. Rev. D 73, 123504 (2006), arXiv:astro-ph/0511218.

[17] Y.-S. Song, I. Sawicki, and W. Hu, Phys. Rev. D 75, 064003 (2007).

[18] F. Schmidt, M. Liguori, and S. Dodelson, Phys. Rev. D 76, 083518 (2007), arXiv:0706.1775.

[19] H. Oyaizu, M. Lima, and W. Hu, Phys. Rev. D 78, 123524 (2008), 0807.2462.

[20] F. Schmidt, M. Lima, H. Oyaizu, and W. Hu, ArXiv e-prints (2008), 0812.0545.

[21] G. Dvali, G. Gabadadze, and M. Porrati, Physics Letters B 485, 208 (2000), arXiv:hep-th/0005016.

[22] A. Nicolis and R. Rattazzi, Journal of High Energy Physics 6, 59 (2004), arXiv:hep-th/0404159.

[23] C. Deffayet, Physics Letters B 502, 199 (2001), arXiv:hep-th/0010186.

[24] M. A. Luty, M. Porrati, and R. Rattazzi, Journal of High Energy Physics 2003, 029 (2003), URL http://stacks.iop. org $/ 1126-6708 / 2003 / i=09 / a=029$.

[25] G. Dvali, New Journal of Physics 8, 326 (2006), arXiv:hep-th/0610013.

[26] K. Koyama, ArXiv e-prints (2007), 0709.2399.

[27] K. Koyama and R. Maartens, Journal of Cosmology and Astro-Particle Physics 1, 16 (2006), arXiv:astro-ph/0511634.

[28] A. Cardoso, K. Koyama, S. S. Seahra, and F. P. Silva, Physical Review D (Particles, Fields, Gravitation, and Cosmology) 77, 083512 (pages 16) (2008), URL http://link.aps.org/abstract/PRD/v77/e083512.

[29] W. Fang, W. Hu, and A. Lewis, Phys. Rev. D 78, 087303 (2008), 0808.3125.

[30] W. Fang, S. Wang, W. Hu, Z. Haiman, L. Hui, and M. May, Phys. Rev. D 78, 103509 (2008), 0808.2208.

[31] G. Dvali, S. Hofmann, and J. Khoury, Phys. Rev. D 76, 084006 (2007), arXiv:hep-th/0703027.

[32] C. de Rham, S. Hofmann, J. Khoury, and A. J. Tolley, Journal of Cosmology and Astro-Particle Physics 2, 11 (2008), 0712.2821 .

[33] N. Afshordi, G. Geshnizjani, and J. Khoury, ArXiv e-prints (2008), 0812.2244.

[34] A. Nicolis, R. Rattazzi, and E. Trincherini, Phys. Rev. D 79, 064036 (2009), 0811.2197.

[35] L. Lombriser, W. Hu, W. Fang, and U. Seljak, ArXiv e-prints (2009), 0905.1112.

[36] K. Koyama and F. P. Silva, Phys. Rev. D 75, 084040 (2007), arXiv:hep-th/0702169.

[37] K. Koyama, A. Taruya, and T. Hiramatsu, ArXiv e-prints (2009), 0902.0618.

[38] J. Khoury and M. Wyman, ArXiv e-prints (2009), 0903.1292.

[39] G. W. Gibbons and S. W. Hawking, Phys. Rev. D 15, 2752 (1977).

[40] A. I. Vainshtein, Physics Letters B 39, 393 (1972), ISSN 0370-2693.

[41] C. Deffayet, G. Dvali, G. Gabadadze, and A. Vainshtein, Phys. Rev. D 65, 044026 (2002), arXiv:hep-th/0106001.

[42] H. Oyaizu, Phys. Rev. D 78, 123523 (2008), 0807.2449.

[43] R. W. Hockney and J. W. Eastwood, Computer Simulation Using Particles (Computer Simulation Using Particles, New York: McGraw-Hill, 1981, 1981).

[44] A. Klypin and J. Holtzman, ArXiv Astrophysics e-prints (1997), astro-ph/9712217.

[45] A. Brandt, Math. Comput. 31, 333 (1977).

[46] A. Brandt, Tech. Rep. (GMD-Studien) Nr. 85, Gesellschaft für Mathematik und Datenverarbeitung, Schloss Birlinghoven, Sankt Augustin, Germany (1984).

[47] W. L. Briggs, V. E. Henson, and S. F. McCormick, A multigrid tutorial (2nd ed.) (Society for Industrial and Applied Mathematics, Philadelphia, PA, USA, 2000), ISBN 0-89871-462-1.

[48] W. Fang, S. Wang, W. Hu, Z. Haiman, L. Hui, and M. May, Phys. Rev. D 78, 103509 (2008), 0808.2208. 
[49] A. Lewis, A. Challinor, and A. Lasenby, Astrophys. J. 538, 473 (2000), astro-ph/9911177.

[50] R. E. Smith, J. A. Peacock, A. Jenkins, S. D. M. White, C. S. Frenk, F. R. Pearce, P. A. Thomas, G. Efstathiou, and H. M. P. Couchman, MNRAS 341, 1311 (2003), arXiv:astro-ph/0207664.

[51] J. L. Tinker, A. V. Kravtsov, A. Klypin, K. Abazajian, M. S. Warren, G. Yepes, S. Gottlober, and D. E. Holz, ArXiv e-prints 803 (2008), 0803.2706.

[52] I. Laszlo and R. Bean, Phys. Rev. D 77, 024048 (2008), 0709.0307.

[53] S. D. M. White, J. F. Navarro, A. E. Evrard, and C. S. Frenk, Nature (London) 366, 429 (1993).

[54] V. R. Eke, S. Cole, C. S. Frenk, and J. Patrick Henry, MNRAS 298, 1145 (1998), arXiv:astro-ph/9802350.

[55] S. Borgani, P. Rosati, P. Tozzi, S. A. Stanford, P. R. Eisenhardt, C. Lidman, B. Holden, R. Della Ceca, C. Norman, and G. Squires, Astrophys. J. 561, 13 (2001), arXiv:astro-ph/0106428.

[56] A. Vikhlinin, A. V. Kravtsov, R. A. Burenin, H. Ebeling, W. R. Forman, A. Hornstrup, C. Jones, S. S. Murray, D. Nagai, H. Quintana, et al., Astrophys. J. 692, 1060 (2009), 0812.2720.

[57] A. Lue, R. Scoccimarro, and G. D. Starkman, Phys. Rev. D 69, 124015 (2004), arXiv:astro-ph/0401515.

[58] D. J. Eisenstein, I. Zehavi, D. W. Hogg, R. Scoccimarro, M. R. Blanton, R. C. Nichol, R. Scranton, H.-J. Seo, M. Tegmark, Z. Zheng, et al., Astrophys. J. 633, 560 (2005), arXiv:astro-ph/0501171.

[59] W. J. Percival, S. Cole, D. J. Eisenstein, R. C. Nichol, J. A. Peacock, A. C. Pope, and A. S. Szalay, MNRAS 381, 1053 (2007), 0705.3323.

[60] W. Hu, private communication.

[61] L. Fu, E. Semboloni, H. Hoekstra, M. Kilbinger, L. van Waerbeke, I. Tereno, Y. Mellier, C. Heymans, J. Coupon, K. Benabed, et al., A\&A 479, 9 (2008), 0712.0884.

[62] URL http://fftw.org/.

[63] S. F. Shandarin, Astrophysics 16, 439 (1980).

[64] A. V. Kravtsov, A. A. Klypin, and A. M. Khokhlov, Astrophys. J. Supplement 111, 73 (1997), arXiv:astro-ph/9701195.

[65] W. Hu and I. Sawicki, Phys. Rev. D 76, 104043 (2007), arXiv:0708.1190. 